\title{
Exercise-Induced Engagement of the IL-15/IL15Ra axis Promotes Anti-Tumor Immunity
}

\section{Dafna Bar-Sagi ( $\sim$ Dafna.Bar-Sagi@nyumc.org )}

New York University School of Medicine https://orcid.org/0000-0003-2597-8948

\section{Emma Kurz}

New York University School of Medicine

\section{Tanner Dalton}

Columbia University Irving Medical Center

\section{Sorin Shadaloey}

New York University School of Medicine

\section{Emily Vucic}

New York University School of Medicine

\section{Rafael Winograd}

New York University Langone Health

\section{Carolina Hirsch}

New York University School of Medicine

\section{Article}

Keywords: Tumor Infiltration, Aerobic Exercise, Murine Models, Pancreatic Cancer, Epinephrine, PD-1 Blockade

Posted Date: January 18th, 2021

DOI: https://doi.org/10.21203/rs.3.rs-130050/v1

License: (c) (1) This work is licensed under a Creative Commons Attribution 4.0 International License. Read Full License 


\section{Exercise-Induced Engagement of the IL-15/IL15R $\alpha$ axis Promotes Anti-Tumor Immunity}

4 Authors: Emma Kurz ${ }^{\wedge}$, Carolina Alcantara Hirsch ${ }^{1,3}$, Tanner Dalton ${ }^{2}$, Sorin Alberto Shadoloey ${ }^{1}$, 5 Emily Vucic $^{3}$, Rafael Winograd ${ }^{4}$, Dafna Bar-Sagi ${ }^{3 *}$

6 Affiliations:

$7{ }^{1}$ Department of Cell Biology, NYU Grossman School of Medicine, $5501^{\text {st }}$ Avenue, New York, 8 NY, 10016

$9{ }^{2}$ Department of Cell Biology, Columbia University Irving Medical Center, 630 W 168th St. New 10 York, NY, 10032

$11{ }^{3}$ Department of Biochemistry and Molecular Pharmacology, NYU Grossman School of Medicine, $125501^{\text {st }}$ Avenue, New York, NY, 10016

$13{ }^{4}$ Permultter Cancer Center, NYU Langone Health, 160 East 34 ${ }^{\text {th }}$ Street, New York, NY, 10016

$14 \quad\left(^{*}\right)$ symbol indicates corresponding author information. $\left(^{\wedge}\right)$ indicates first authorship.

15 Corresponding author contact: dafna.bar-sagi@nyulangone.org

One Sentence Summary: Exercise induces tumor protection and anti-tumor immunity in PDA.

\section{$\underline{\text { Abstract }}$}

Tumor-infiltrating immune cells play a central role in controlling cancer development and progression, as well as in responses to therapeutic interventions. However, the mechanisms that control their mobilization, composition, and function are not completely understood. Here, we show that aerobic exercise is sufficient to induce an intra-tumoral expansion of activated CD8 T cells and a reduction in tumor growth in murine models of pancreatic cancer. Specifically, exercise-induced spikes in epinephrine promote a systemic immune mobilization and accumulation of tumor-infiltrating IL15R $\alpha+$ CD8 T cells. This sub-population of activated CD8 T cells is responsible for the tumor protective and immune activating benefits of aerobic exercise, as both are abrogated in the context of IL-15 antagonism. Notably, the anti-tumor effect of aerobic exercise is potentiated by PD-1 blockade, suggesting a therapeutically exploitable link between an exercise-oncology axis and immune intervention strategies in a largely intractable disease. 
MAIN TEXT

35 A growing body of evidence indicates that frequent structured exercise is associated with 36 decreased incidence of cancer and cancer-associated mortalities ${ }^{1,2,3,4,5}$. Preclinical studies utilizing

37 murine models of breast cancer, liver cancer, and melanoma have implicated modulation of tumor 38 vascularity, sex/growth hormone signaling, metabolic alterations, and immune cell engagement as 39 potential mechanisms underlying the tumor-protective effects of exercise $e^{6,7,8,9}$. Despite these 40 advances, little is known about the anti-tumor effects of exercise in the context of pancreatic ductal 41 adenocarcinoma (PDA), a deadly disease for which current therapeutic options are woefully 42 limited. In the present study we sought to identify local and systemic factors that could precipitate 43 exercise-dependent tumor protective effects in pancreatic cancer.

\section{Routine aerobic exercise restricts pancreatic tumor growth}

46 To assess the impact of aerobic exercise on pancreatic tumor growth, we subjected animals to a 47 mild intensity forced treadmill-running exercise regimen [5x/week; $30 \mathrm{mins} ; 15 \mathrm{~cm} / \mathrm{sec}]^{10,11}$, 48 hereafter referred to as aerobic exercise (Schematic Figure 1a). In a slow-progressing 49 autochthonous genetic model of PDA [p48-Cre;LSL-KRas ${ }^{\mathrm{G} 12 \mathrm{D}} / \mathrm{KC}$ mice], aerobic exercise led to 50 a pronounced delay in disease development, as evidenced by preservation of the acinar 51 compartment and an associated reduction in desmoplastic reaction and metaplastic conversion

52 (Figure 1b, Extended Data 1a). We next examined the effect of aerobic exercise on tumor growth 53 in an orthotopic model of PDA, wherein wild-type (WT) mice are subjected to intra-pancreatic 54 injection of $\mathrm{p} 53^{\mathrm{R} 172 \mathrm{H} / \mathrm{t}}-\mathrm{KRAS}^{\mathrm{G} 12 \mathrm{D}}$ (KPC) mutant cells and are sacrificed 3-4 weeks post-operation 55 (post-op) $^{12}$. In this model, aerobic exercise resulted in a reduction in tumor weight when initiated 56 on post-op Day 1 concurrent with tumor implantation (Figure 1c, Extended Data 1b), or on 57 post-op Day 12, when mice bear already-established tumors (Figure 1d). Notably, prolonging the 58 duration (time) or increasing the intensity (speed) of aerobic exercise did not result in an additional 59 degree of tumor protection in the KPC orthotopic model (Extended Data 1c). In aggregate, these 60 findings indicate that aerobic exercise provides tumor protective benefits in the setting of both 61 pancreatic tumor initiation and disease progression. 
63 Exercise-mediated tumor protection requires CD8 T cells

64 Aerobic exercise has been known to modulate immune function and immune cell mobilization ${ }^{13}$,

6514 . The potential relevance of these modulatory effects to exercise-induced tumor protection is 66 suggested by the loss of protection in athymic nude (Figure 2a) or Rag1KO mice (Extended Data 67 2a). As these results indicate an essential role for mature lymphocytes, we performed an unbiased 68 analysis of the intra-tumoral immune milieu using single cell RNA sequencing (sc-RNA seq). 69 Comparison of leukocytes isolated from exercise and control orthotopic KPC tumors revealed an 70 approximate five-fold expansion of CD8 $\mathrm{T}$ cells (Cluster 1) in the tumors of exercised mice 71 (Figure $\mathbf{2 b}, \mathbf{2 g}$ ). This increase in the number of CD8 T cells was corroborated using multiplex 72 immune-fluorescence microscopy (Figure 2c). Subsequent analysis of the gene expression profile 73 of the expanded CD8 $\mathrm{T}$ cell (Cluster 1) population demonstrated a distinctively activated and 74 cytotoxic phenotype, evidenced by high expression of Gzmk, Gzmb, Icos, CD28, and Ccl5 75 (Extended Data 2b). Upstream canonical pathway analysis also showed a significant upregulation 76 of a cytotoxic/anti-tumor program in this population (Figure 2d). Using flow cytometry, we confirmed that intra-tumoral CD8 $\mathrm{T}$ cells isolated from exercised animals bear a distinctive cytotoxic and activated phenotype as compared to control animals (Figure 2e, Gating Strategy

79 Extended Data 2c). Based on these observations, we hypothesized that this population is essential for exercise-mediated reduction in tumor growth in PDA. To test this hypothesis, CD8 T cells were

81 depleted in control and exercised mice by the serial administration of a targeted depleting antibody 82 (Extended Data 2d). In the absence of CD8 T cells, no reduction in tumor growth was observed 83 in exercised mice (Figure 2f), indicating the essentiality of CD8 T cell expansion and activation 84 in exercise-induced tumor protection.

86 In addition to activated CD8 T cells (Cluster 1), the most prevalent immune populations detected 87 in both exercise and control tumors on sc-RNA seq included exhausted CD8 T cells (Cluster 2), 88 myeloid suppressor cells (Cluster 3), CD4 T cells (Cluster 4), NK cells (Cluster 5), and B cells 89 (Cluster 6) (Figure 2b). With the exception of a notable decrease in the fraction of myeloid 90 suppressor cells (Cluster 3), no change in the relative fraction of intra-tumoral immune cells that 91 comprise these clusters was observed between exercise and control tumors (Figure 2g). The 92 mechanisms underlying the exercise-dependent decrease in the abundance of myeloid suppressor 93 cells remains to be determined. However, the contribution of this change to the anti-tumor effect 
94 of exercise likely reflects a decrease in myeloid-derived immune tolerant signals upstream of 95 effector $\mathrm{T}$ cells. This immunologic hierarchy has been previously reported in PDA ${ }^{15,16,17}$ and is

96 consistent with our data documenting the complete dependency of exercise-induced tumor 97 protection on activated CD8 T cells (Figure 2f).

Exercise-induced increase of intra-tumoral CD8 $T$ cells is dependent on peripheral

100 lymphocyte mobilization

101 Aerobic exercise-induced leukocytosis (EIL) and subsequent redistribution is a phenomenon

102 mediated, in part, through exercise-associated spikes in epinephrine (Epi) that mobilize various 103 immune populations to peripheral blood ${ }^{18,19,20}$. The contribution of EIL to our observed exercise104 induced intra-tumoral immune alterations is suggested by the doubling of serum Epi levels 105 detected at 30 minutes post-exercise with concomitant fractional increases in peripheral and intra106 tumoral T cells (Figure 3a-b). This conclusion is further supported by the finding that treatment 107 of exercised mice with non-selective beta-adrenergic blocker propranolol showed a reversal of 108 both tumor protection and CD8 T cell expansion and activation (Figure 3c-d). Based on these 109 findings, we sought to determine whether the increase in peripheral blood lymphocytes is required 110 for exercise-mediated intra-tumoral CD8 T cell expansion. To this end, we utilized Fingolimod 111 (FTY720), a drug known to induce marked lymphopenia via inhibition of lymphocyte egress from 112 thymus or secondary lymphoid organs (SLO) ${ }^{21,22,23}$. Consistent with its mode of action, FTY720 113 abrogated the increase in total T cells and CD8 T cells in the blood of exercised mice (Extended 114 Data 3a,c). Of note, FTY720 treatment also reversed exercise-induced increases in intra-tumoral 115 CD8 T cells and abrogated exercise-mediated tumor protection (Figure 3e-f, Extended Data 3b). 116 These findings implicate both adrenergic-mediated-EIL and peripheral migration of $\mathrm{T}$ cells as 117 essential facilitators of the tumor protective and immune-modulatory effects of exercise in this 118 disease model.

\section{$\underline{\text { IL15/IL15R } \alpha \text { axis is required for exercise-induced tumor protection }}$}

121 The role of IL-6, IL-8, and IL-15, cytokines that are upregulated during strenuous exercise, in 122 mediating the metabolic and immune responses to tissue damage has been well established ${ }^{24,25}$. Of 123 the above, only free IL-15 and secreted IL-15/IL15r $\alpha$ complexes (IL-15 bound to the alpha subunit 124 of IL-15 receptor) have been shown to promote CD8 $\mathrm{T}$ cell survival and induce a cytotoxic 
125 phenotype through signal transduction in a cis or trans manner, respectively ${ }^{26,27,28}$. As we 126 consistently observed a cytotoxic CD8 T cell phenotype in the tumors of exercised mice, we set 127 out to test whether IL-15 responsive cells may contribute to exercise-induced anti-tumor immunity. 128 Using flow cytometry and multiplex immune-fluorescence microscopy, we observed a significant increase in the fraction and number of IL15R $\alpha+$ CD8 T cells in the tumors of exercised mice, with no change in IL15R $\alpha+$ CD4 T cells (Figure 4a-b). Phenotypic analysis of the tumor-infiltrating

131 IL15R $\alpha+$ subset of CD8 $\mathrm{T}$ cells in exercised mice showed a significant upregulation of 132 proliferation and activation markers compared to their IL15R $\alpha$ negative counterparts, consistent 133 with engagement of IL-15R signaling (Figure 4c). The essentiality of this axis was further 134 established by demonstrating that treatment of exercised mice with an IL-15 neutralizing antibody 135 was sufficient to reverse both exercise-mediated tumor protection and intra-tumoral increase of 136 activated IL15R $\alpha+$ CD8 T cells (Figure 4d-f, Extended Data Figure 3d). These data support a 137 role for the IL-15/IL15R $\alpha+$ axis in exercise-mediated intra-tumoral immune activation.

139 We next addressed the question of whether IL-15R $\alpha+$ CD8 T cells, akin to their parent population, 140 may undergo beta-adrenergic-dependent peripheral mobilization via EIL. To this end, mice were 141 sacrificed 30 minutes after completion of exercise and the levels of IL-15R $\alpha+$ CD8 T cells in the 142 periphery were assayed. Consistent with an EIL-based mobilization mechanism observed for 143 exercise-induced increase in intra-tumoral activated CD8 T cells (Fig 3d-e), exercise induced an 144 increase in the number of IL-15R $\alpha+$ CD8 T cells in peripheral blood (Extended Data 3e), and this 145 effect was mimicked by the administration of a physiological dose of epinephrine, albeit on a 146 smaller scale (Figure 4g). In addition, treatment of mice with the beta-blocker propranolol 147 inhibited the exercise-induced increase of IL-15R $\alpha+$ CD8 T cells in the tumor, indicating 148 adrenergic-mediated mobilization of this cell population (Figure 4h). Furthermore, based on an 149 earlier study reporting IL-15 production in tumors ${ }^{29}$, we performed an analysis of levels of IL-15 150 in KPC orthotopic tumors and found it is produced by both immune and non-immune cellular 151 compartments (Figure 4i), suggesting the possibility that IL15R $\alpha+$ CD8 T cells may be engaged 152 by their cognate cytokine in the PDA microenvironment. Collectively, these observations support 153 a role for both systemic and localized engagement of the IL-15/IL-15R $\alpha+$ signaling axis in 154 mediating exercise-induced anti-tumor immunity and protection. 
157 Given our observations that intra-tumoral CD8 T cells, including IL-15R $\alpha+$ CD8 T cells display 158 selectively high expression of the exhaustion marker PD-1 in exercised animals (Figure 2e, 159 Extended Data 4a), we tested whether the immune-modulatory effects of exercise could be 160 enhanced when combined with $\alpha$-PD-1. Treatment of mice with a PD-1 blocking antibody over 161 the course of aerobic exercise was accompanied by a global enhancement of exercise-dependent 162 anti-tumor modulatory effects, evidenced by increases in the total number of CD3+ $\mathrm{T}$ cells 163 (Extended Data 4b), CD8 T cells (Figure 5a), and the proliferative, activated, and cytotoxic 164 phenotype of this latter population (Figure 5b). In addition, we observed an increase in the number

165 of tumor-infiltrating IL-15R $\alpha+$ CD8 $\mathrm{T}$ cells in exercised mice treated with $\alpha$-PD-1, suggesting 166 that these cells may be responsive to checkpoint blockade (Figure 5c, Extended Data 4c). 167 Importantly, combination of exercise and $\alpha$-PD-1 led to a significantly more effective reduction in 168 tumor growth compared to exercise alone (Figure 5d), suggesting that PD-1 blockade could 169 enhance the tumor-protective and immune-activating efficacy of aerobic exercise in pancreatic 170 cancer.

\section{Discussion}

173 Our findings uncover a hitherto unknown link between exercise-induced immune cell mobilization 174 and pancreatic tumor protection involving IL-15 signaling and cytotoxic $\mathrm{T}$ cell-mediated 175 anti-tumor immunity (Figure 5e). The consistency of the tumor-protective effects observed across multiple experimental murine models of pancreatic tumorigenesis suggests a broadly applicable association between aerobic exercise and tumor control in pancreatic cancer. Furthermore, the

178 finding that even mild exercise is sufficient to profoundly alter the intra-tumoral immune milieu points to the potential utility and accessibility of physical-activity-based interventions for pancreatic cancer patients, a population with significant morbidities ${ }^{30}$.

182 To date, the impact of aerobic exercise on immune cell redistribution and activation has been 183 shown to be principally mediated by circulating serum cytokines or altered systemic metabolites 184 that directly promote immune cell activation in the perpihery ${ }^{6,9}$. Our findings add a new dimension 185 to the immune-modulatory effects of aerobic exercise by implicating the pancreatic tumor itself as 186 a site of immune cell activation through the production of IL-15 by tumor and stromal cells. 
187 Furthermore, the failure of exercise to induce protection in a syngeneic sub-Q model of PDA

188 (Extended Figure 1d) suggests that a cross-talk between systemic EIL and an organ-specific 189 tumor microenvironment may constitute an important determinant of the protective benefits of 190 exercise.

192 In conclusion, our work demonstrates that exercise alone can prime the pancreatic tumor 193 microenvironment for improved responsiveness to immune-based therapeautics. This insight 194 should provide the basis for future combinatorial approaches involving aerobic exercise and 195 immune-modulatory agents in treatment-resistant and immune-supressed solid tumors like 196 pancreatic cancer.

198 STAR Methods:

199 Murine exercise, disease models, and in-vivo treatment regimens

200 C57BL/6, Rag1KO, and FoxNude athymic mice were purchased from Jackson Labs (Bar Harbor, $201 \mathrm{ME}$ ) and bred in-house. LSL-KRAS ${ }^{\mathrm{G} 12 \mathrm{D} /+} ; \mathrm{p}^{2} 8^{\mathrm{Cre} /+}(\mathrm{KC})$ mice were bred in the Division of 202 Comparative Medicine mouse facility at New York University Langone Medical Center. Both 203 male and female mice were used, as indicated, and animals were age matched within each 204 experiment. For orthotopic studies, 8-10 week old mice were administered intra-pancreatic

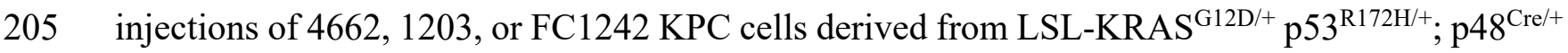
206 (KPC) mice, as previously described ${ }^{31}$. Briefly, cells were suspended in PBS with 50\% Matrigel 207 (BD Biosciences, Franklin Lakes, NJ) and either $5 \times 10^{4}$ or $1 \times 10^{5}$ KPC cells were injected into the 208 pancreas via laparotomy. Mice were sacrificed on Day 21 for analyses, unless otherwise indicated.

209 For subcutaneous studies, $1 \times 10^{6}$ KPC cells were implanted into the flank of age-matched mice and 210 mice were sacrificed at Day 33 or upon tumor ulceration. For all experiments, unless otherwise 211 indicated, exercised mice were involuntarily placed on a Rodent 5-lane treadmill (Harvard 212 Apparatus, Cat No: 76-0895), for 30 minutes per day at $15 \mathrm{~cm} / \mathrm{second}$, for a minimum of 5 213 days/week. In the 3 days leading up to sacrifice, exercise mice were obligately exercised, 214 regardless of previous number of consecutive days exercised. Mice that were unable to complete 215 treadmill running routine exercise were removed from the experiment. For initial experiments, 216 sham control mice in matched experiments were placed on a stationary $(0 \mathrm{~cm} / \mathrm{sec})$ treadmill for 217 the same duration of time as experimental mice. After ten independent experimental repeats using 
218 sham controls, experiments were performed with control mice remaining in their cage when no 219 difference in sham control and control mice in the cage were observed. Where indicated, 220 neutralizing antibodies directed against CD8 (200 $\mu$ g, clone 2.43), IL-15 (200 $\mu \mathrm{g}$, clone AIO.3), 221 or PD-1 (200 $\mu \mathrm{g}$, clone RMP1-14) were utilized (all from BioXcell, West Lebanon, NH) 3x weekly 222 using regimens previously described ${ }^{32,33,34}$. Where indicated, mice were treated every day 223 intraperitoneally (i.p.) with $2 \mathrm{mg} / \mathrm{kg}$ with FTY720 (Sigma Aldrich). Stocks of FTY720 were 224 resuspended in DMSO $(20 \mathrm{mg} / \mathrm{mL})$ and stored at $-80^{\circ} \mathrm{C}$; . for injection, FTY720 was first 225 resuspended in 2\% Bhydroxypropyl-cyclodextrin in PBS and diluted at $45 \%$ volume in PBS 226 (Sigma Aldrich) based on previous protocols ${ }^{35}$. Where indicated, mice were provided either 227 normal drinking water or $.5 \mathrm{~g} / \mathrm{L}$ propranolol (Sigma Aldrich) drinking water and bifurcated into 228 control and exercise cohorts ${ }^{36}$. All studies were approved by the Institutional Animal Care and Use 229 Committee at NYU School of Medicine. Experiments were conducted in accordance with the NYU 230 School of Medicine policies on the care, welfare, and treatment of laboratory animals. All 231 experiments met or exceeded the standards of the Association for the Assessment and 232 Accreditation of Laboratory Animal Care, International (AAALAC), the United States Department 233 of Health and Human Services, and all local and federal animal welfare laws.

\section{Cellular Preparation, Flow Cytometry, and FACS}

236 Single cell suspensions of PDA tumors were prepared for flow cytometry as described previously 237 with slight modifications ${ }^{17}$. Briefly, pancreata were placed in cold 2\% FACS (cold PBS with 2\%

238 FBS) with Collagenase IV (1 mg/mL; Worthington Biochemical, Lakewood, NJ), Trypsin 239 inhibitor (1mg/mL; EMD Millipore, Billerica, MA) and DNase I (2 U/mL; Promega, Madison, $240 \mathrm{WI}$ ), and minced with scissors to sub-millimeter pieces. Tissues were then incubated at $37^{\circ} \mathrm{C}$ for 24120 minutes with gentle shaking every 5 minutes and then passed through a $70 \mu \mathrm{m}$ mesh and 242 centrifuged at $350 \mathrm{~g}$ for 5 minutes. Cell pellets were re-suspended in FACS. After blocking 243 FcrRIII/II with an anti-CD16/CD32 mAb (eBiosciences, San Diego, CA), cells were labeled by

244 incubating $1 \times 10^{6}$ cells with $1 \mu \mathrm{g}$ of fluorescently conjugated mAbs directed against mouse CD44 245 (IM7), PD-1 (29F.1A12), CD3 (17A2), CD4 (RM4-5), CD8 (53-6.7), CD45 (30-F11), CD11b 246 (M1/70), Gr1 (RB6-8C5), MHC II (M5/114.15.2), IFN $\gamma$ (XMG1.2), ICOS (15F9), CD69 247 (H1.2F3), IL-15ralpha (6B4C88), Ki67 (16A8), CD140a (APA5), EPCAM (G8.8), CD34 248 (MEC14.7), (all BioLegend, San Diego, CA), IL-15 (Thermo Fisher, clone 34559), T-bet 
(eBio4B10), and Granzyme B (NGZB). For flow cytometry of whole blood, PBMC were isolated

250 by overlaying whole blood diluted 1:1 in PBS over an equal amount of Ficoll (GE Healthcare,

251 Princeton, NJ). Cells were then spun at 2100 RPM and the buffy coat harvested as described ${ }^{37}$.

252 Dead cells were excluded from analysis using zombie yellow (BioLegend). Flow cytometry was

253 performed on the Attune NxT Flow Cytometer (ThermoFisher, Waltham, MA). FACS-sorting was

254 performed on the SY3200 (Sony, Tokyo, Japan). Data were analyzed using FlowJo Version 10.6.1

255 (Treestar, Ashland, OR).

Histology, Immunofluorescence, and Microscopy

258 Tissues were fixed for 48 hours in $10 \%$ buffered formalin at $4^{\circ} \mathrm{C}$ and embedded into paraffin in a

259 Leica Peloris automated processor. Five-micron sections of the paraffin-embedded tissues were 260 stained with hematoxylin and eosin (H\&E) or Gomori Trichrome, where appropriate. Histology 261 was analyzed on a Zeiss LSM700 confocal microscope. The percentage of acinar area (fibrosis) in 262 each slide was calculated on Adobe Photoshop software by dividing the number of pancreatic 263 acinar pixels (or Trichrome Gomore blue stained pixels) over the total number of tissue pixels 264 present in each field of view (FOV). Where appropriate, FFPE samples were stained with Akoya 265 Biosciences ${ }^{\circledR}$ Opal $^{\mathrm{TM}}$ multiplex automation kit (Akoya Biosciences, Menlo Park, CA). Automated 266 staining was performed on Leica BondRX ${ }^{\circledR}$ autostainer (Leica Microsystems, Inc., Buffalo Grove, 267 IL). The protocol was performed according to manufacturers' instructions. Primary antibodies 268 included CD3 (Biorad, cat \# MCA1477T), CD8 (Cell Signaling Tech, cat \# 98941), IL15ra 269 (ThermoFisher, cat \# PA5-79467) and CK8 (TROMA-I) [TROMA-I, deposited to the DSHB by 270 Brulet, P. / Kemler, R. (DSHB Hybridoma Product TROMA-I)]. Briefly, all slides underwent 271 sequential epitope retrieval, antibody incubation and tyramide signal amplification (TSA). Primary 272 and secondary antibodies were removed during epitope retrieval steps while fluorophores remain 273 covalently attached to the epitope. Multispectral Image Acquisition and Analysis was performed 274 on a Vectra ${ }^{\circledR}$ Polaris multispectral imaging system (Akoya Biosciences, Menlo Park, CA) and the 275 fluorophores spectrally unmixed using either Phenochart (for whole slide scans) or InForm (for 276 selected MSI fields) software. 
278 In indicated experiments, wild type mice were injected i.p. with $20 \mu \mathrm{g}$ of Epinephrine dissolved in

$279200 \mu \mathrm{l}$ of PBS and sacrificed 30 minutes after injection. $100 \mu \mathrm{l}$ of whole blood was collected,

280 processed, and assessed by flow cytometry. In separate experiments, mice were subjected to 30

281 minutes of exercise, then sacrificed 20 minutes after completion of exercise, and $200 \mu 1$ of whole

282 blood was collected. Sera was isolated from whole blood using a previously described 283 centrifugation isolation ${ }^{38}$, and subjected to Epinephrine ELISA detection kit (Novus Biologicals, 284 CO).

285

286 Single Cell RNAseq Data Pre-Processing

287 Sequencing results were demultiplexed and converted to FASTQ format using Illumina bcl2fastq 288 software. The Cell Ranger Single-Cell Software Suite (https://support.10xgenomics.com/single289 cell-gene-expression/software/pipelines/latest/what-is-cell-ranger) was used to perform sample 290 demultiplexing, barcode processing, and single-cell 3' gene counting. The cDNA insert was 291 aligned to the mm10/GRCm38 reference genome. Only confidently mapped non-PCR duplicates 292 with valid barcodes and UMIs were used to generate the gene-barcode matrix. Further analysis 293 including the identification of highly variable genes, dimensionality reduction, standard 294 unsupervised clustering algorithms, and the discovery of differentially expressed genes was 295 performed using the Seurat R package ${ }^{39,40}$. To exclude low quality cells, cells that were extreme 296 outliers in terms of library complexity, or cells that may possibly be multiple cells or doublets, we 297 calculated the distribution of genes detected per cell and removed any cells in the top and bottom $2982 \%$ quantiles. We additionally removed cells with more than $10 \%$ of the transcripts coming from 299 mitochondrial genes.

Integrated Analysis of Single Cell Datasets

302 To account for technical batch differences between the three libraries, we utilized the Seurat 303 alignment method for data integration, which specifically does not expect that confounding 304 variables have uniform effects on all cells in a dataset and allows for global transcriptional shifts 305 between datasets. Seurat uses a variant of canonical correlation analysis (CCA) to find linear 306 combinations of features and identifies shared correlation structures across datasets. For each 307 dataset, we identified variable genes, while controlling for the strong relationship between 308 variability and average expression. We took the union of the top 2,000 genes with the highest 
dispersion from both datasets and ran a CCA to determine the common sources of variation

310 between datasets. We then aligned the subspaces based on the first 15 canonical correlation

311 vectors, generating a new dimensionality reduction that was then used for further analysis. For

312 single cell analysis of an unbiased pool of $\mathrm{CD} 45^{+}$tumor-infiltrating leukocytes, we refined our

313 analysis to include $\mathrm{CD} 45^{+}$expressing cells that expressed $>500$ detected genes. We then

314 normalized the data by the total expression, multiplied this by a scale factor of 10,000 , and log-

315 transformed the result. The final dataset included 4,216 cells with a median of 1,103 detected

316 genes.

Visualization and Clustering of Single Cell RNAseq Data

319 To visualize the data, we further reduced the dimensionality of the dataset to project the cells in two-dimensional space using PCA followed by t-distributed Stochastic Neighbor Embedding (tSNE) based on the aligned CCA. Aligned CCA was also used as a basis for partitioning the 322 dataset into clusters using a smart local moving (SLM) community detection algorithm 323 (https://arxiv.org/ftp/arxiv/papers/1308/1308.6604.pdf). To find markers that define individual 324 clusters, we performed differential expression analysis using Wilcoxon rank sum test for each 325 cluster compared to all other cells for genes detected in at least $20 \%$ of the cluster cells. The initial analysis of PDA-infiltrating leukocytes yielded 16 clusters. We assigned cell type identities based on the expression of known population markers as follows: Cluster 1: CD8+ T cells $-C D 8 a^{h i}$

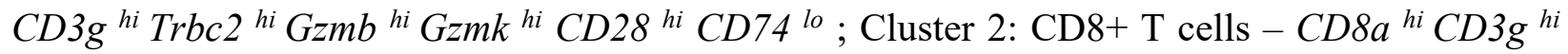
Trbc1 ${ }^{\text {hi }} \mathrm{Gzmb}^{\text {lo }} \mathrm{Gzmk}^{\text {lo }} \mathrm{Pdcd} 1^{\text {hi }} \mathrm{Lag} 3^{\text {hi}}$; Cluster 3: Myeloid Suppressor Cells- Hdc ${ }^{\text {hi }}$ Mmp9 ${ }^{\text {hi }}$

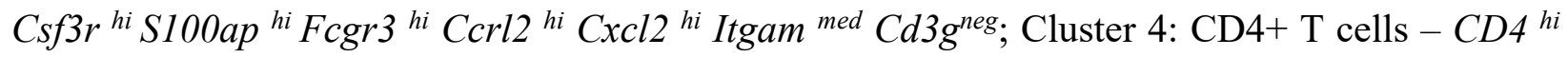
Icos ${ }^{h i} C D 28^{h i} C D 3 g^{h i}$ Trbc2 ${ }^{h i} C D 8 a^{l o}$; Cluster 5: NK Cells - Klrblc ${ }^{\text {hi }}$ Klra8 ${ }^{\text {hi }}$ Klra9 ${ }^{\text {hi }}$ Gzma ${ }^{\text {hi }}$ Klrc2 ${ }^{\text {hi }}$ Trbc2 ${ }^{\text {lo }}$ Eomes $^{\text {hi }}$ Ncr1 ${ }^{\text {hi }}$ Trbc2 ${ }^{\text {lo }}$; Cluster 6: B Cells - CD79a ${ }^{\text {hi }}$ Iglc2 ${ }^{\text {hi }}$ CD79b ${ }^{\text {hi }}$ Ebf1 ${ }^{\text {hi }}$ CD19 hi ${ }^{\text {Igkc }}{ }^{\text {hi }}$ Trbc2 ${ }^{\text {lo }} \mathrm{Nkg} 7^{\text {lo }} \mathrm{CD} 22^{\text {hi }}$; Cluster 7: CD4- CD8- T cells - CD4 ${ }^{\text {neg }}$ CD8a ${ }^{\text {lo }}$ Lef1 hi

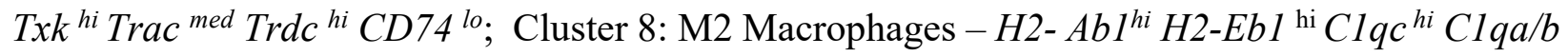
${ }^{h i}$ Arg1 ${ }^{h i} \mathrm{Mmp12} / 13^{\text {hi }}$ Apoe ${ }^{\text {hi }} \mathrm{CD} 68^{\text {hi }} \mathrm{CD} 74^{\text {hi }} \mathrm{Nkg} 7^{\text {lo }} \mathrm{CD} 8 \mathrm{b1}{ }^{\mathrm{lo}}$; Cluster 9: Dendritic Cells (P1) Clec 9a ${ }^{\text {hi }}$ Itgae $^{\text {hi }}$ Batf3 ${ }^{\text {hi PPt1 }}{ }^{\text {hi }}$ Plet1 ${ }^{\text {hi }}$ Ifi205 ${ }^{\text {hi }}$ Flt3 ${ }^{\text {hi }}$ Tlr $3^{\text {hi }}$; Cluster 10: Monocyte/Macrophages

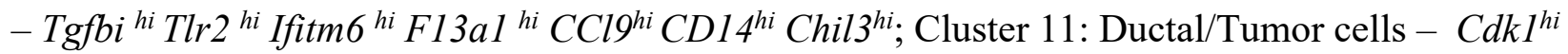
Mki67 $^{\text {hi }} \mathrm{Cdkn}^{\text {hi }} \mathrm{Cit}^{\text {hi }} \mathrm{Cdca} 8^{\text {hi } C C n b 2}{ }^{\text {hi }}$; Cluster 12: Dendritic Cells (L) - Ffar2 ${ }^{\text {hi }}$ Epcam $^{\text {med }}$ Siglece ${ }^{\text {med }}$ Batf3 ${ }^{\text {med }} \mathrm{Mgl2}^{\text {hi }} \mathrm{Kmo}^{\text {hi }}$; Cluster 13: CD209 ${ }^{\text {hi }}$ Myeloid Cells $-C D 209 a^{\text {hi CD209d }}{ }^{\text {hi }}$ Ddr $1^{\text {hi }}$ 


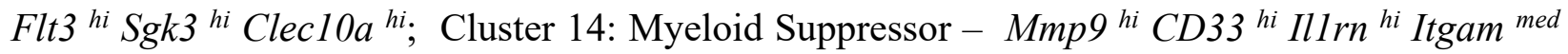
Cxcr2 ${ }^{\text {hi }}$ Trem $1{ }^{\text {hi }} \mathrm{CD} 14{ }^{\text {hi }}{ }^{\text {Pilra }}{ }^{\text {hi }} \mathrm{Csf} 3 \mathrm{rr}^{\text {hi }} \mathrm{Cxcl} 2^{\text {hi }} \mathrm{Hdc}{ }^{\text {hi }}$; Cluster 15: Dendritic Cells (L2) - Cacnb3

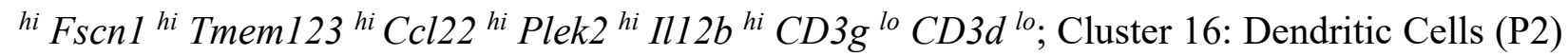
- Siglech ${ }^{\text {hi Lairl }}{ }^{\text {hi }} \mathrm{Upb1}{ }^{\text {hi }} \mathrm{Klk1}{ }^{\text {hi }}{ }^{\mathrm{C} D} 3 d^{\text {lo }}{ }^{\mathrm{CD}} 3 \mathrm{~g}{ }^{l o}$. The raw gene counts data were used for

344 further differential expression analysis. To identify the differentially expressed genes, Seurat R package was used. The resulting genes with adjusted $\mathrm{p}<0.05$ were considered significant. To identify the signaling pathways in which genes are enriched, Ingenuity Pathway Analysis was carried out for genes that were considered significant. The canonical pathways analyzed in IPA software (Ingenuity Pathway Analysis, Qiagen) are represented as bar-plots.

Statistical Analysis

Data is presented as mean $+/$ - standard error. Statistical significance was determined by the Student's $t$ test using GraphPad Prism 7, where indicated (GraphPad Software, La Jolla, CA). pvalues $<0.05$ were considered statistically significant.

Author Contributions:

(EK) - experimental design, manuscript preparation, project oversight, and experimental execution. (CAH) - experimental execution and design. (TD) experimental design and execution. (SAS) - experimental execution. (EV) - manuscript preparation and experimental design. (RW) manuscript preparation and experimental design. (DBS) - experimental design, manuscript preparation, project oversight.

364 The KPC cell line (line 4662) was a kind gift from Dr. R.H. Vonderheide. We would like to thank 365 the New York University Langone Medical Center (NYULMC) Genome Technology Center 366 (GTC) and Flow Cytometry Core each supported in part by the Laura and Isaac Perlmutter Cancer 367 Center Support Grant P30CA016087 and by grant UL1 TR000038 from the National Center for 368 the Advancement of Translational Science (NCATS). We specifically thank members of the 369 Experimental Pathology Research Laboratory, which is partially supported by NIH/NCI 5 370 P30CA16087 (VM \& CL) and S10 OD021747 (PerkinElmer/AkoyaBiosciences ${ }^{\circledR}$ Vectra ${ }^{\circledR}$ 371 multispectral imaging system), for their technical support and expertise. E.K. was supported by 
372 NIH/NCI grant F30 CA243205 and T32- GM136573. E.A. Vucic was supported by a Canadian 373 Institutes of Health Research Fellowship (146792). This work was supported by NIH/NCI Grant 374 CA210263 (D.B.-S).

375

376

377

378

379

380

381

382

383

384

385

386

387

388

389

390

391

392

393

394

395

396

397

398

399

400

401

402 
Figure 1

a

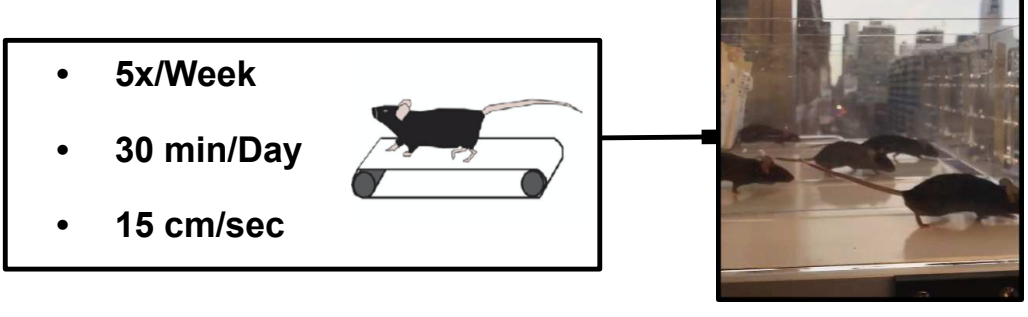

b
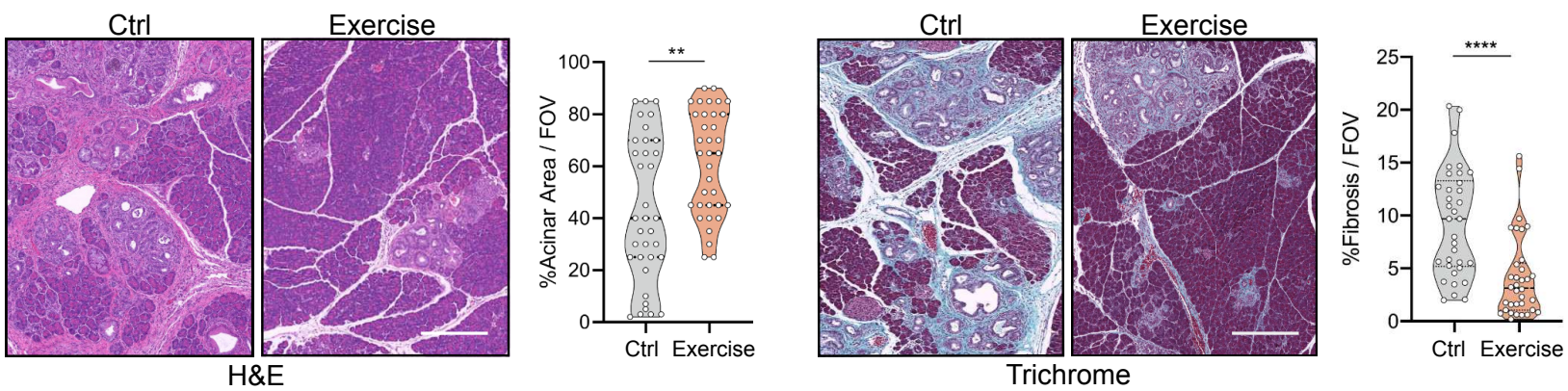

C

d
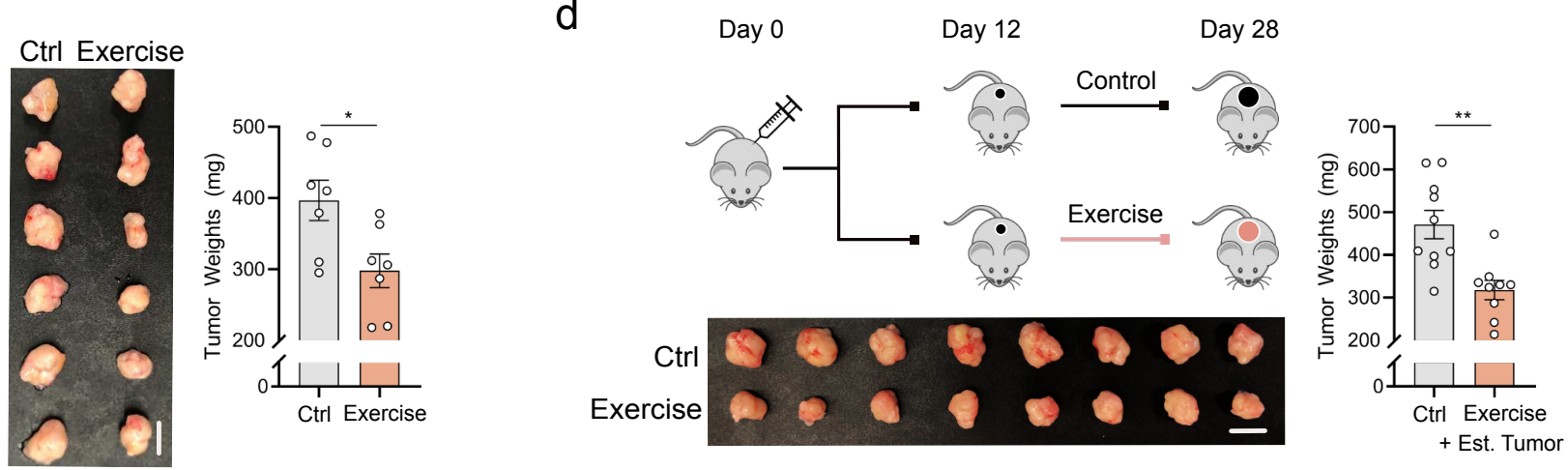

Figure 1: Aerobic exercise restricts pancreatic cancer growth and reduces tumor size

a. The forced treadmill running model (aerobic exercise) is shown. The frequency, speed, and

406 duration of aerobic exercise are indicated (left). Representative photograph of mice running on the

407 treadmill is shown (right). b. 8-week old LSL-KRAS ${ }^{\mathrm{G} 12 \mathrm{D} / \mathrm{WT}}$; p48 $8^{\mathrm{Cre} / \mathrm{WT}}$ (KC) mice were exercised

408 (Exercise) for 6 weeks. Control mice remained sedentary. Mice were sacrificed at 14 weeks old.

409 Histologic staining of pancreata tissue sections with Hematoxylin and Eosin (H\&E, left) and

410 Trichrome and Gomori (Trichrome, right) are shown. Representative images and quantification of

411 acinar (left) and fibrotic (right) areas are shown. Each dot represents quantification in one field of

412 view (FOV; 5-9 FOV analyzed from 3 tissue sections separated by at least $100 \mu \mathrm{m} ; \mathrm{n}=5$ mice).

413 Scale bar represents $200 \mu$ m. c. 8-week old female C57BL/6J wild type mice (WT) were injected

414 orthotopically with $1 \times 10^{\wedge}$ LSL-KRAS $^{\mathrm{G} 12 \mathrm{D} / \mathrm{WT}}$; LSL-Trp53 $172 \mathrm{H} / \mathrm{WT}$; p48 ${ }^{\mathrm{Cre} / \mathrm{WT}}$ (KPC) 4662 cells

415 into the pancreas at Day 0 and exercise was started at Day 1. Control mice remained sedentary.

416 Mice were sacrificed at Day 21. Representative tumor images (left) and quantification of tumor 
417 weights (right) are shown. Scale bar represents $1 \mathrm{~cm}$. Each dot represents one tumor $(\mathrm{n}=7)$. d. 8418 week old female WT mice were injected orthotopically with $5 \times 10^{\wedge} 4$ KPC 4662 cells into the 419 pancreas at Day 0. At Day 12 following implantation, mice were randomized into control and 420 exercise cohorts. Mice were sacrificed at Day 28. Schematic of experimental design (top), tumor 421 images (bottom) and quantification of tumor weights (right) are shown. Scale bar represents $1 \mathrm{~cm}$. 422 Each dot represents one tumor $(\mathrm{n}=9-10) .(\mathrm{p}<0.05=*, \mathrm{p}<0.001=* *, \mathrm{p}<0.0001=* * * *)$. 


\section{Figure 2}

a
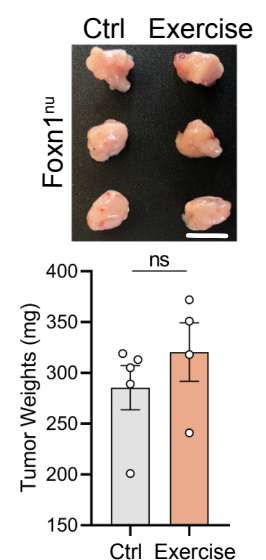

b
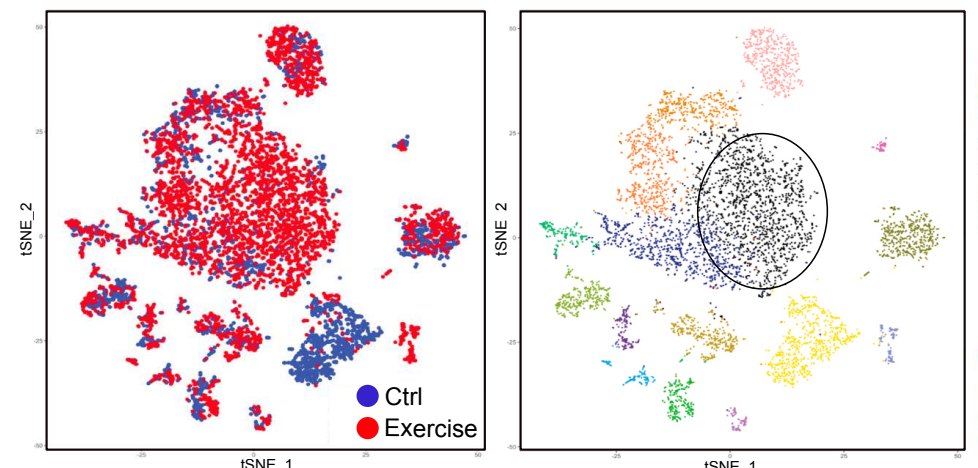

CD8+ $T$ cells $[1]$

CD8+ $T$ cells [2]

Myeloid Suppressor [3]

CD4+ T cells $[4]$

NK Cells [5]

B cells [6]

CD4- CD8- T cells [7]

M2 Macrophages [8]

Dendritic Cells (P1)[9]

Monocyte/Macrophages [10]

Ductal/Tumor Cells [11]

Dendritic Cells (L) [12]

CD209hi Myeloid [13]

Myeloid Suppressor [14]

Dendritic Cells (L2) [15]

C
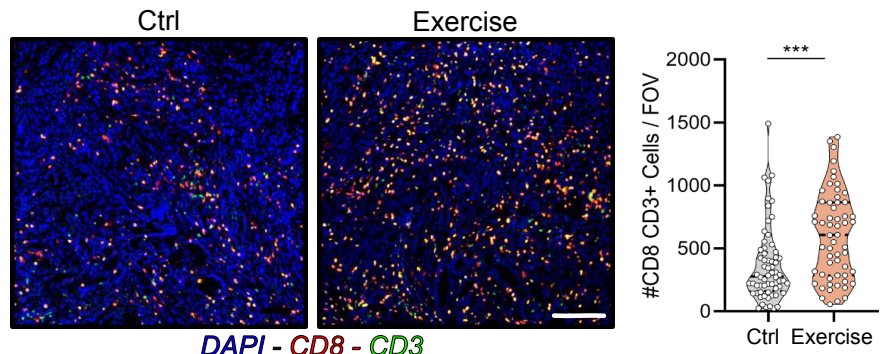

d

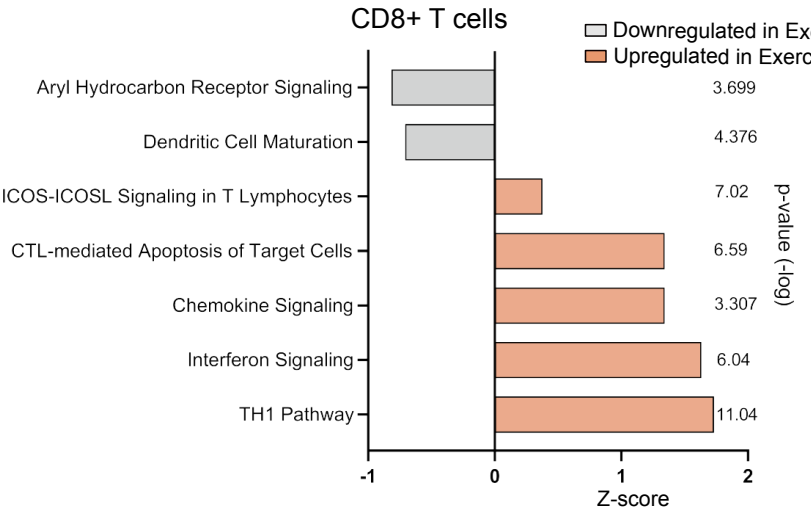

e

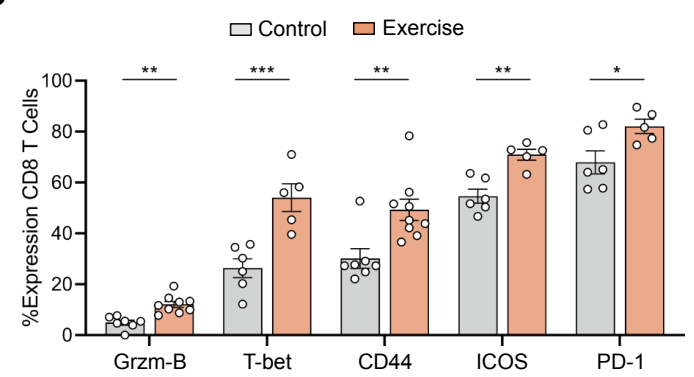

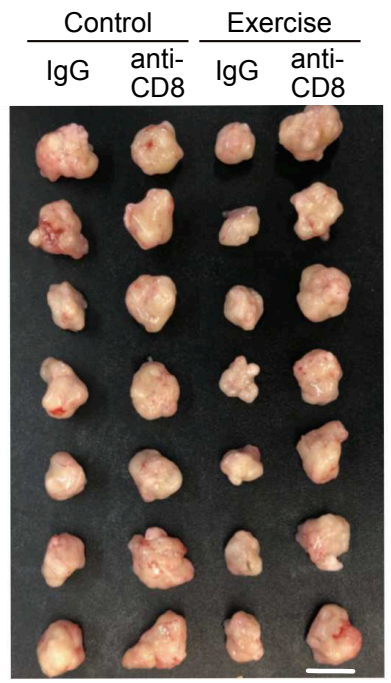
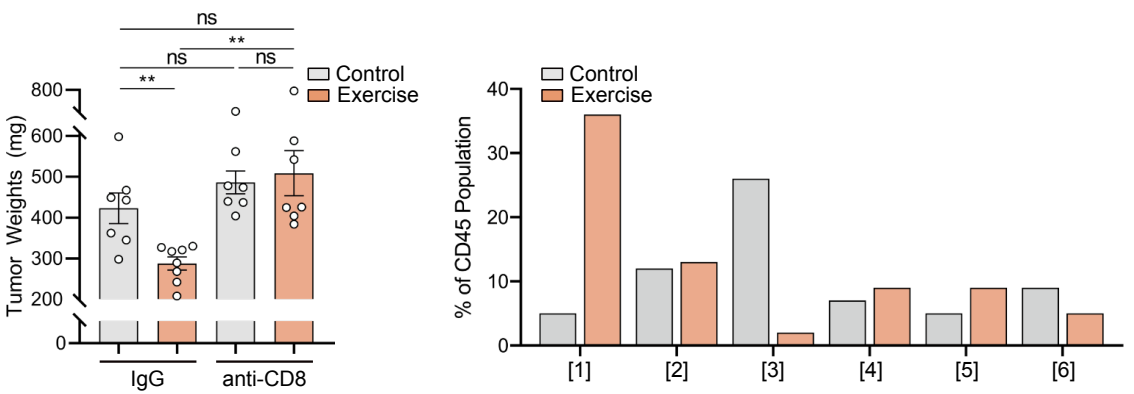
450 a. 8 week old female athymic nude NU/J mice were injected orthotopically with $1 \times 10 \wedge 5$ KPC 4662 451 cells at Day 0 and exercise was started at Day 1. Control mice remained sedentary. Mice were 452 sacrificed at Day 21. Representative tumor images (top) and quantification of tumor weights 453 (below) are shown. Scale bar represents $1 \mathrm{~cm}$. Each dot represents one tumor $(\mathrm{n}=4-5)$. b-g. 8454 week old female C57BL/6J wild type mice (WT) were injected orthotopically with $1 \times 10^{\wedge} \mathrm{KPC}$ 4554662 cells into the pancreas at Day 0 and exercise was started at Day 1. Control mice remained 456 sedentary. Mice were sacrificed at Day 21 and tumors analyzed as follows. b. Single cell RNASeq 457 (scRNAseq) was performed on live leukocytes (PI-, CD45+) isolated by FACS. The distribution 458 of cellular clusters was determined using the tSNE algorithm: control (blue) and exercise (red) 459 leukocytes were identified (left), phenotypic clusters were identified by distinct colors (middle) 460 and labeled (right) ( $\mathrm{n}=3$ tumors pooled in each group). c. Tumor sections were stained by 461 multiplex immunofluorescence for CD8 (red), CD3 (green), and DAPI (blue). The number of $462 \mathrm{CD} 8+\mathrm{CD} 3+$ (yellow) cells were quantified in ImageJ. Each dot represents quantification in one 463 field of view (13-16 FOV analyzed; $n=4)$. Scale bar represents $75 \mu \mathrm{m}$. d. Upstream canonical 464 pathway perturbations were derived using ingenuity pathway analysis. Pathways up-regulated 465 (orange) or down-regulated (grey) in exercise were identified in the CD8+ T cell, Cluster 1. e. 466 Single cell suspensions derived from tumors were stained with antibodies against CD45, CD3, 467 CD4, CD8, Granzyme-B, T-bet, CD44, ICOS, and PD-1 and analyzed by flow cytometry. Each 468 dot represents one tumor $(n=6)$. f. Control and exercise mice were treated $3 \mathrm{x} /$ week with isotype or $469200 \mu \mathrm{g}$ of $\alpha$-CD8 blocking antibody starting on Day 1. Tumor images (left) and quantification of 470 tumor weights (right) are shown. Scale bar represents $1 \mathrm{~cm}$. Each dot represents one tumor $(\mathrm{n}=7)$. 471 g. Quantification of the relative proportion of the six most prominent immune populations in 472 control and exercise tumors from scRNAseq. ( $\mathrm{p}>0.05=\mathrm{ns}, \mathrm{p}<0.05=*, \mathrm{p}<0.01=* *, \mathrm{p}<0.001$ $473=* * *)$. 
Figure 3

a

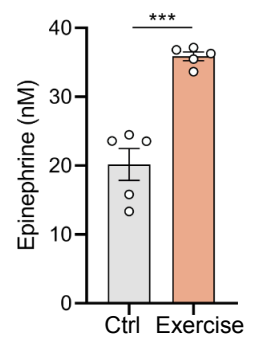

b

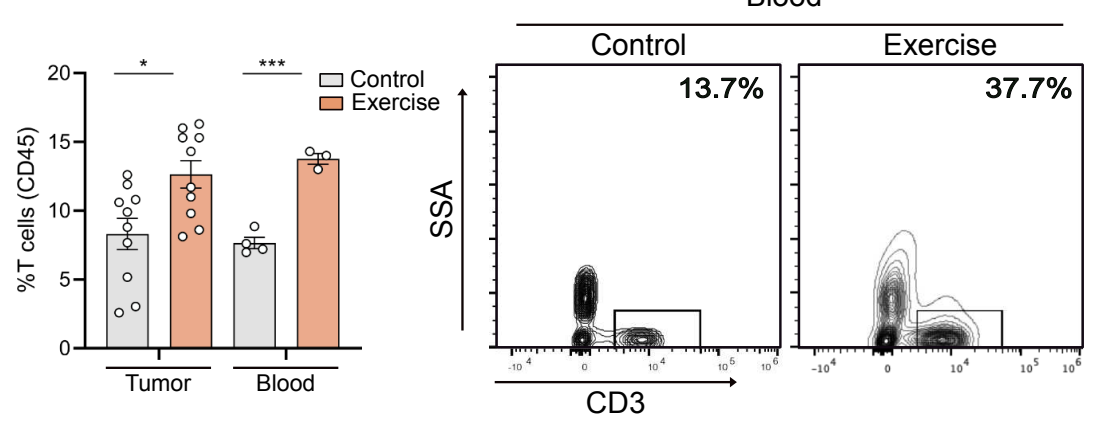

C

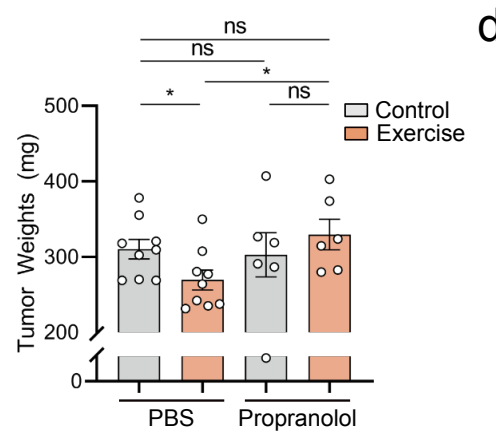

e

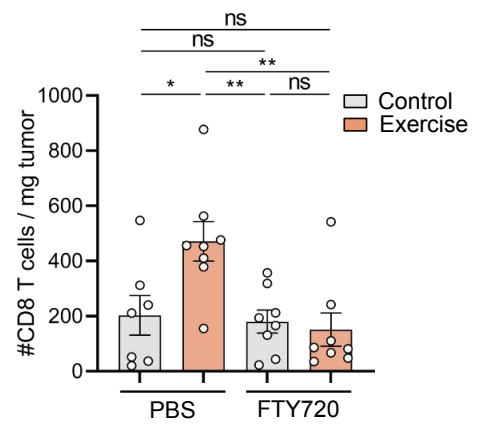

d
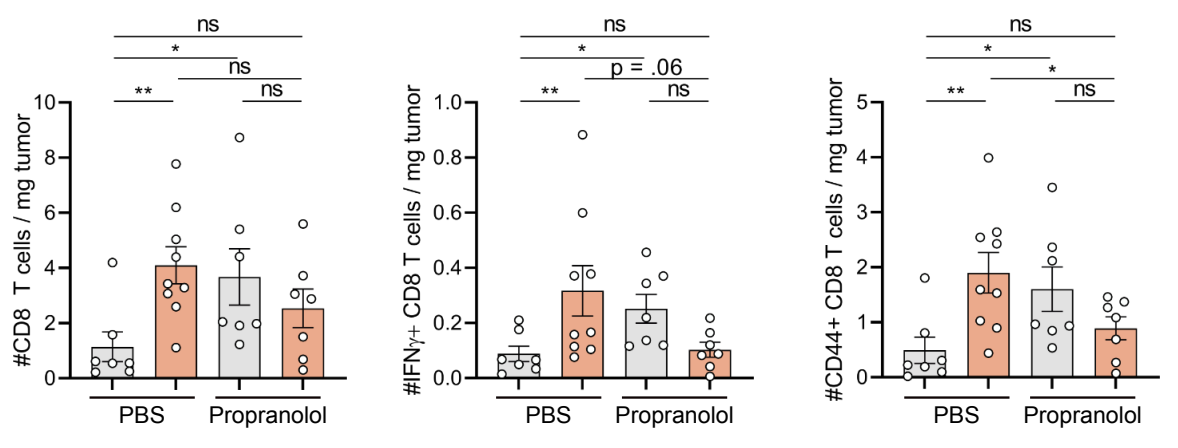

$\square$ Control $\square$ Exercise
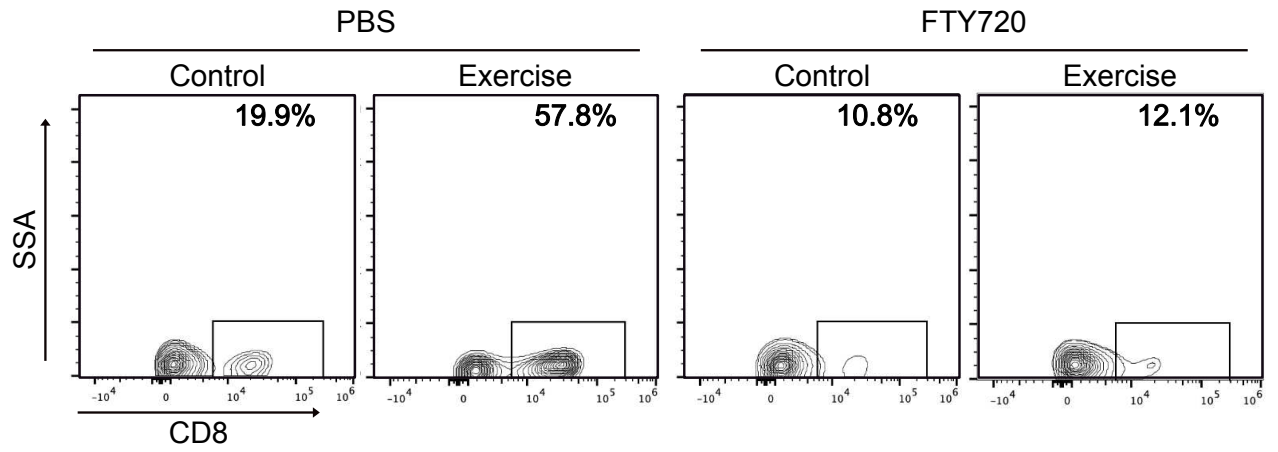
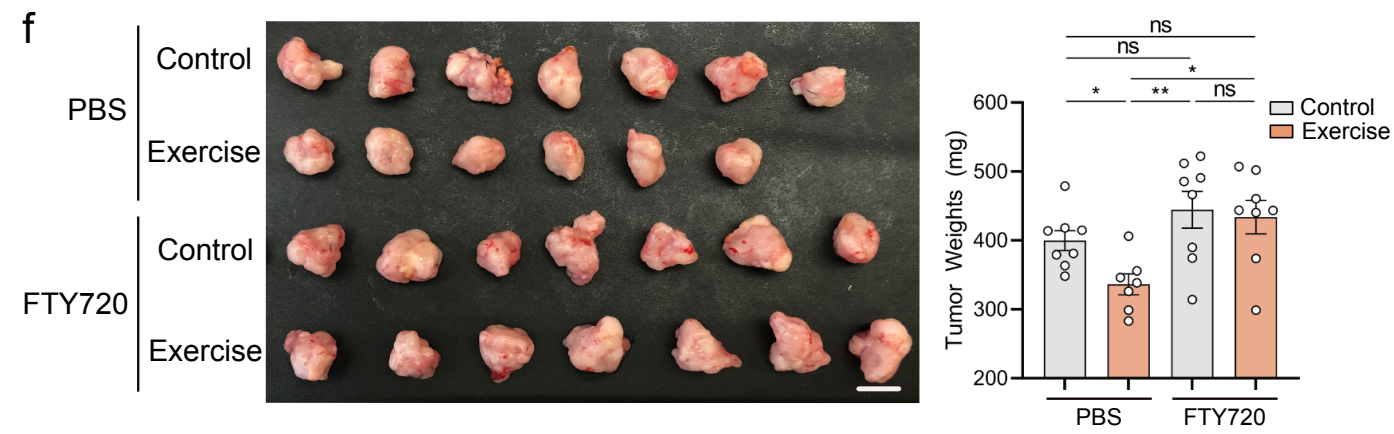

480

481

482

483 
Figure 3: Exercise-induced increase in intra-tumoral CD8 T cells is $\beta$-adrenergic and S1P485 gradient dependent.

486 a. 8 week-old female WT mice were exercised for 30 minutes at $15 \mathrm{~cm} / \mathrm{sec}$ and were sacrificed 20 487 minutes after completion of exercise. $200 \mu$ of whole blood was collected, sera were isolated using 488 centrifugation, and samples were subjected to ELISA for detection of Epinephrine levels. Each dot 489 represents one mouse $(\mathrm{n}=5)$. b-f. 8-week old female C57BL/6J wild type mice $(\mathrm{WT})$ were injected 490 orthotopically with $1 \times 10^{\wedge}$ KPC 4662 cells into the pancreas at Day 0 and exercise was started at 491 Day 1. Control mice remained sedentary. b. Mice were sacrificed at Day 21, thirty minutes after 492 completion of exercise, and tumors and whole blood were harvested, digested, and assessed for 493 relative numbers of CD3 + T cells. Each dot represents one mouse $(n=3-4$ each for blood, $n=10$ 494 for tumors). Representative contour plots are shown for CD3+ cells as a percentage of CD45+ 495 cells in whole blood. c-d. Mice were provided with either normal drinking water or $.5 \mathrm{~g} / \mathrm{L}$ 496 propranolol drinking water ad libitum starting on Day 1. Mice were sacrificed on Day 21 and tumor 497 weights were quantified (c), and single cell suspensions derived from tumors were stained with 498 antibodies against CD45, CD3, CD8, IFN $\gamma$, and CD44 and analyzed by flow cytometry (d). Each 499 dot represents one tumor $(n=6-9)$. e-f. Mice were treated daily either with PBS or $200 \mu$ of 500 fingolimod (FTY720) starting on Day 1. Mice were sacrificed at Day 21. Single cell suspensions 501 derived from tumors were stained with antibodies against CD45, TCRB, CD4, and CD8, and 502 analyzed by flow cytometry. Each dot represents one tumor. Representative flow cytometry plots 503 are shown for CD8+ cells as fraction of CD4- CD3+ cells (e). Tumor images (left) and 504 quantification of tumor weights (right) are shown (f). Scale bar represents $1 \mathrm{~cm}$. Each dot 505 represents one tumor $(\mathrm{n}=7)$. $(\mathrm{p}>0.05=\mathrm{ns}, \mathrm{p}<0.05=*, \mathrm{p}<0.01=* *, \mathrm{p}<0.001=* * *)$. 
Figure 4

a

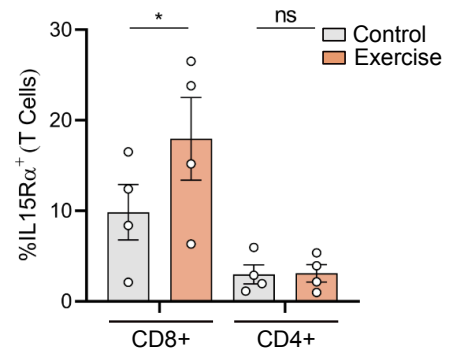

b

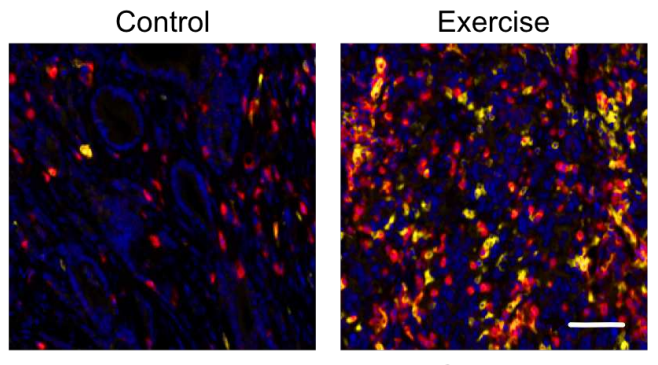

DAPI - IL15Ra - CD8

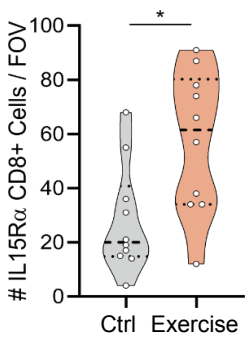

C
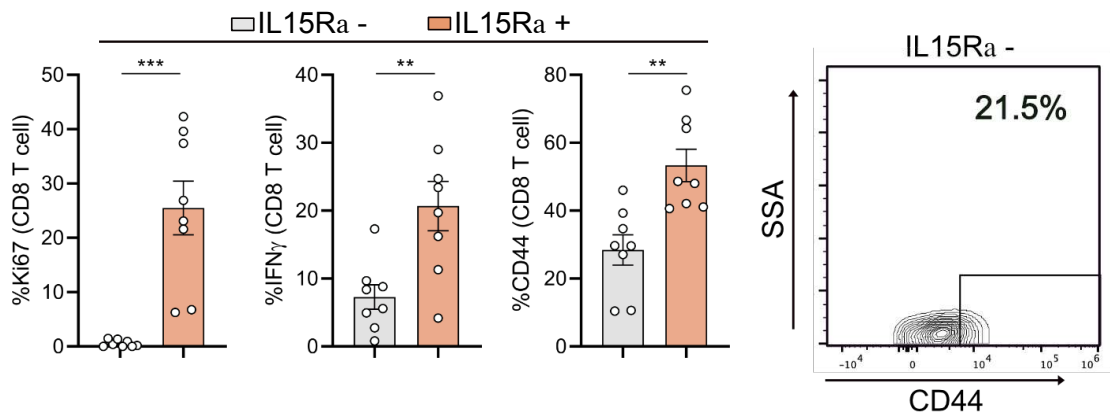

IL15Ra +

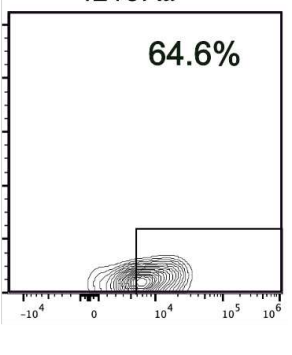

d

$\frac{\lg G}{\text { Ctrl Exercise }} \frac{\text { anti-IL-15 }}{\text { Ctrl Exercise }}$

e

f
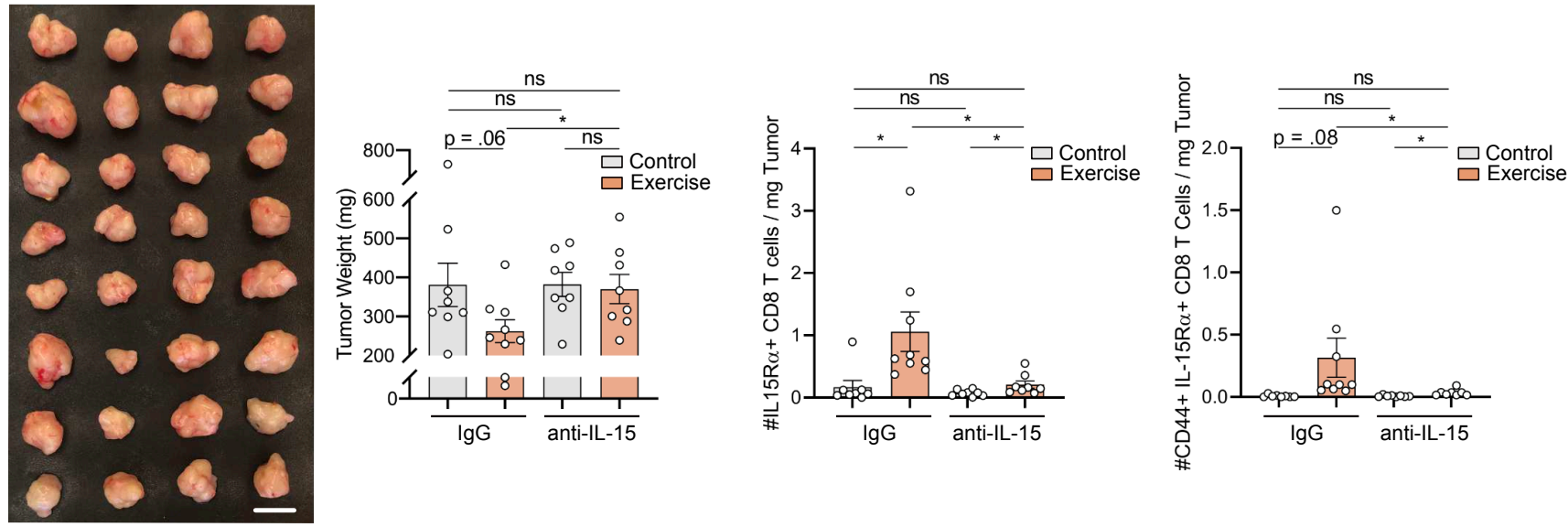

g

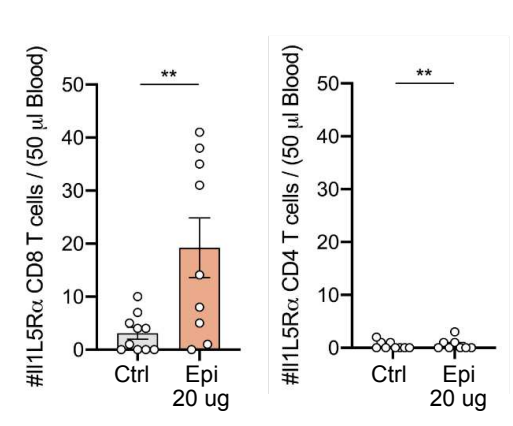

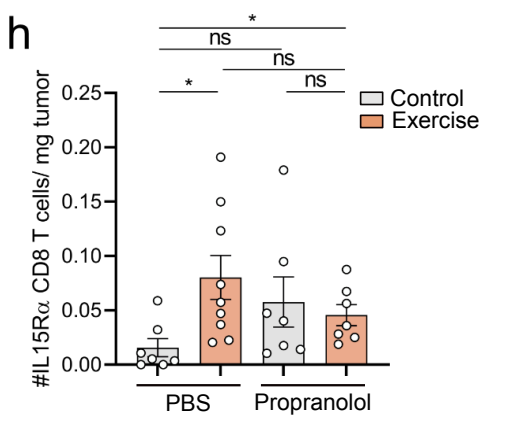

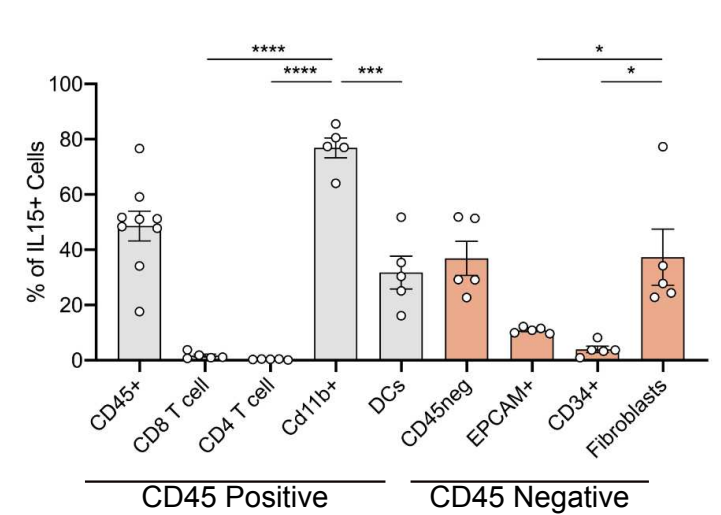




\section{Figure 4: IL-15/IL15Ra axis is required for exercise-mediated tumor protection}

517 a-f. 8-week old female C57BL/6J wild type mice (WT) were injected orthotopically with $1 \times 10^{\wedge 5}$

518 KPC 4662 cells into the pancreas at Day 0 and exercise was started at Day 1. Control mice 519 remained sedentary. Mice were sacrificed at Day 21 and tumors analyzed as follows. a. Single cell 520 suspensions derived from tumors were stained with antibodies against CD45, CD3, CD4, CD8, 521 and IL-15R $\alpha$ and analyzed by flow cytometry. Each dot represents one tumor $(\mathrm{n}=4)$. b. Tumor 522 sections were stained by multiplex immunofluorescence for CD8 (red), IL-15R $\alpha$ (yellow), and 523 DAPI (blue). The number of CD8+ IL-15R $\alpha+$ (orange) cells were quantified in ImageJ. Each dot 524 represents quantification per field of view (3-4 FOV analyzed; $n=3$ ). Scale bar represents $40 \mu \mathrm{m}$. 525 c. Single cell suspensions derived from tumors were stained with antibodies against CD8, Ki67, 526 IFN $\gamma$, CD44 and IL-15R $\alpha$ and analyzed by flow cytometry. Each dot represents one tumor (n=8). 527 Representative flow cytometry plots are shown for CD44. d-f. Mice were treated 3x/week with 528 isotype or $200 \mu \mathrm{g}$ of $\alpha$-IL15 neutralizing antibody starting on Day 1 post-op. Tumor images (left) 529 and quantification of tumor weights (right) are shown. Scale bar represents $1 \mathrm{~cm}(\mathrm{~d})$. Single cell 530 suspensions derived from tumors were stained and analyzed by flow cytometry for number of IL$53115 \mathrm{R} \alpha+\mathrm{CD} 8+\mathrm{T}$ cells (e) and CD44+ IL-15R $\alpha+\mathrm{CD} 8+\mathrm{T}$ cells (f). Each dot represents one tumor 532 (n=8-9). g. 9-week-old female WT mice were injected i.p. with PBS or $20 \mu \mathrm{g}$ of Epinephrine. 533 Thirty minutes post-treatment $100 \mu \mathrm{l}$ of whole blood was isolated and assessed by flow cytometry 534 for number of IL-15R $\alpha+C D 8+T$ cells (left) or IL-15R $\alpha+C D 4+$ T cells (right). Each dot represents 535 one mouse $(\mathrm{n}=9-10)$. h. 8-week old female C57BL/6J wild type mice (WT) were injected 536 orthotopically with $1 \times 10^{\wedge} \mathrm{KPC} 4662$ cells into the pancreas at Day 0 and exercise was started at Day 1. Control mice remained sedentary. Mice were provided either normal drinking water or $538.5 \mathrm{~g} / \mathrm{L}$ propranolol drinking water ad libitum starting on Day 1. Mice were sacrificed at Day 21. 539 Single cell suspensions derived from tumors were assessed by flow cytometry for number of IL$54015 \mathrm{R} \alpha+\mathrm{CD} 8+\mathrm{T}$ cells. Each dot represents one tumor $(\mathrm{n}=6-9)$. i. 8-week old female C57BL/6J 541 wild type mice (WT) were injected orthotopically with $1 \times 10^{\wedge 5} \mathrm{KPC} 4662$ cells into the pancreas at 542 Day 0. All mice remained sedentary. Single cell suspensions derived from tumors were stained 543 with antibodies against CD45, CD3, CD8 T cells (CD8+ CD3+), CD4 T cells (CD4+ CD3+), 544 CD11b, DCs (CD11c+ MHCII hi), EPCAM, CD34, Fibroblasts (CD140a+ CD34- EPCAM545 CD45-) and analyzed by flow cytometry for the expression of IL-15. Each dot represents one tumor 546 $(\mathrm{n}=5-9) .(\mathrm{p}>0.05=\mathrm{ns}, \mathrm{p}<0.05=*, \mathrm{p}<0.01=* *, \mathrm{p}<0.001=* * *, \mathrm{p}<0.0001=* * * *)$. 
Figure 5

a

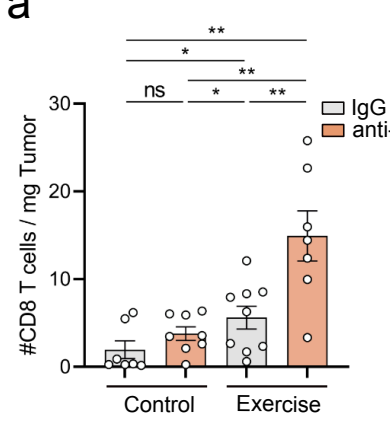

$\lg G$

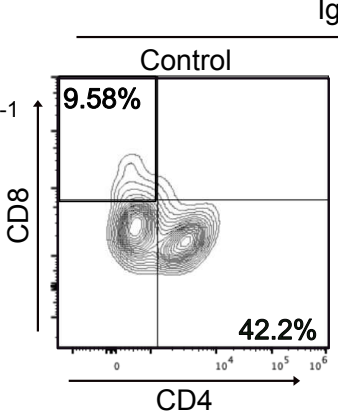

Gxerase

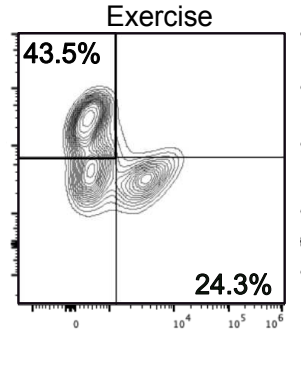

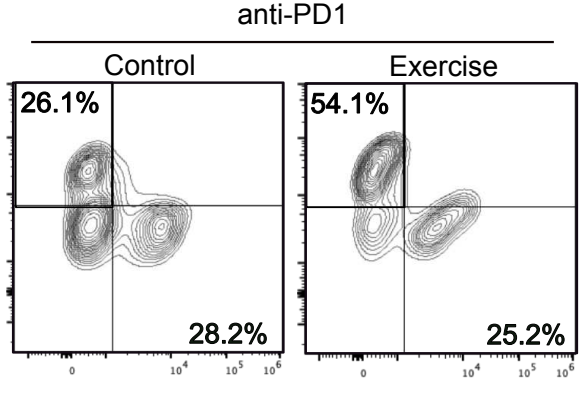

b

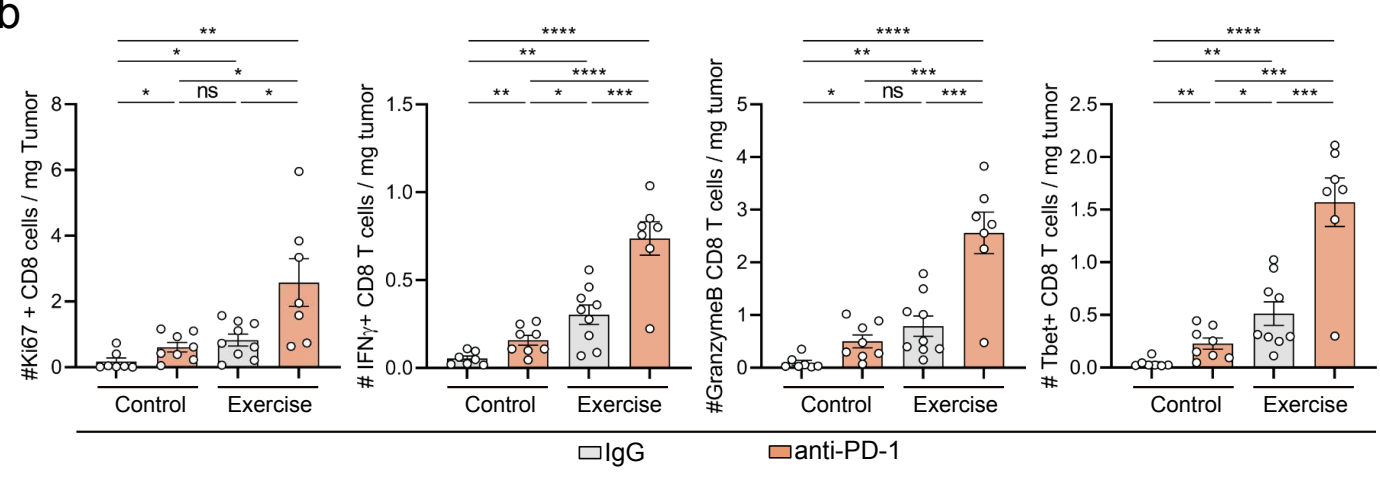

C

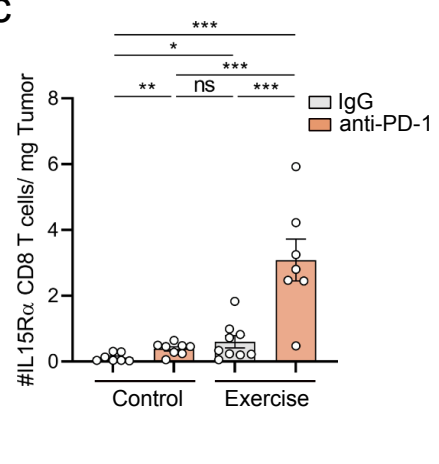

d
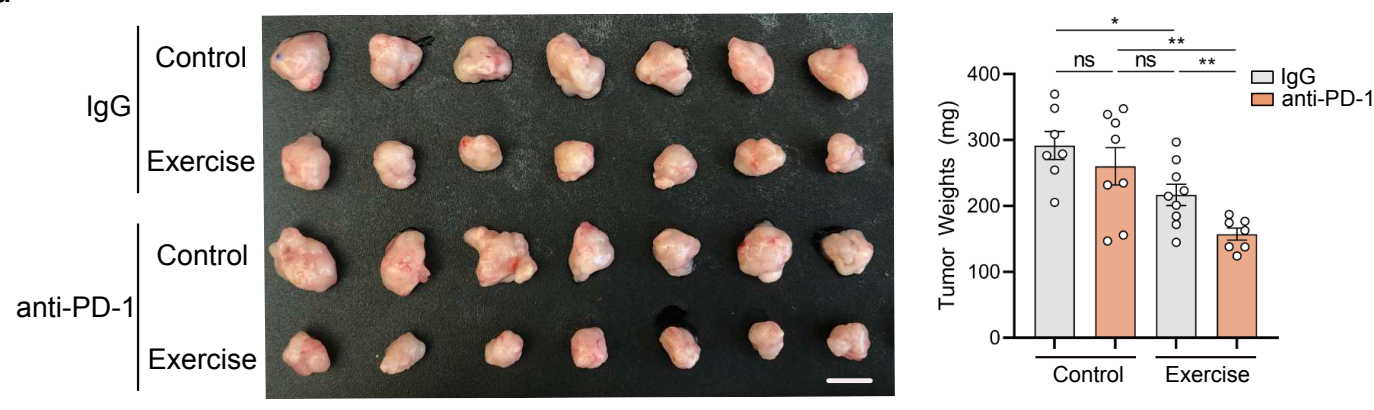

e

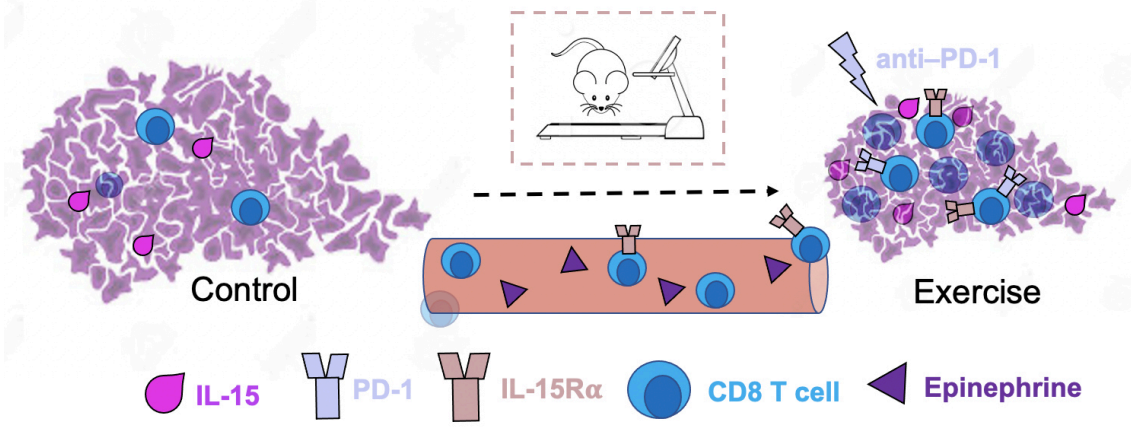

548 Figure 5: Aerobic Exercises Sensitizes Pancreatic Cancer to anti-PD1 Immunotherapy

549 a-d. 8-week old female C57BL/6J wild type mice (WT) were injected orthotopically with $1 \times 10^{\wedge 5}$

550 KPC 4662 cells into the pancreas at Day 0 and exercise was started at Day 1. Control mice 
551 remained sedentary. Mice were treated 3x/week with isotype or $200 \mu \mathrm{g}$ of $\alpha$-PD-1 blocking 552 antibody starting on Day 3. Mice were sacrificed at Day 21. Single cell suspensions derived from 553 tumors were assessed by flow cytometry for CD3, CD4, and CD8 (a), for Ki67, Granzyme-B, T554 bet, and IFN $\gamma$ expression on CD8 T cells (b) and number of IL-15R $\alpha+$ CD8+ T cells (c). Tumor 555 images (left) and quantification of tumor weights (right) are shown (d). Scale bar represents $1 \mathrm{~cm}$. 556 Each dot represents one tumor $(n=7-8)$. e. Schematic depicting proposed mechanism for the impact 557 of aerobic exercise on CD8 T cell mobilization, pancreatic tumor growth and anti-tumor immunity. $558(\mathrm{p}>0.05=\mathrm{ns}, \mathrm{p}<0.05=*, \mathrm{p}<0.01=* *, \mathrm{p}<0.001=* * *, \mathrm{p}<0.0001=* * * *)$. 


\section{Extended Data Figure 1}

a

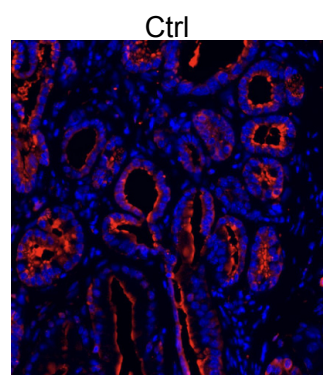

DAPI - CK8
Exercise

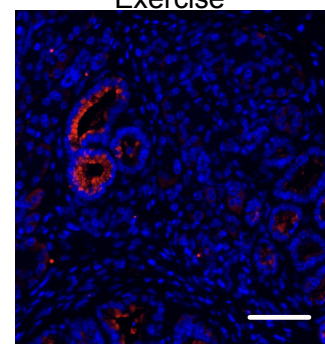

$\longrightarrow$

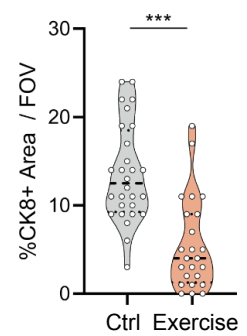

b

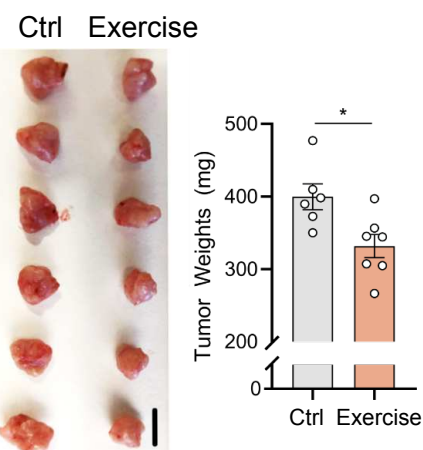

C

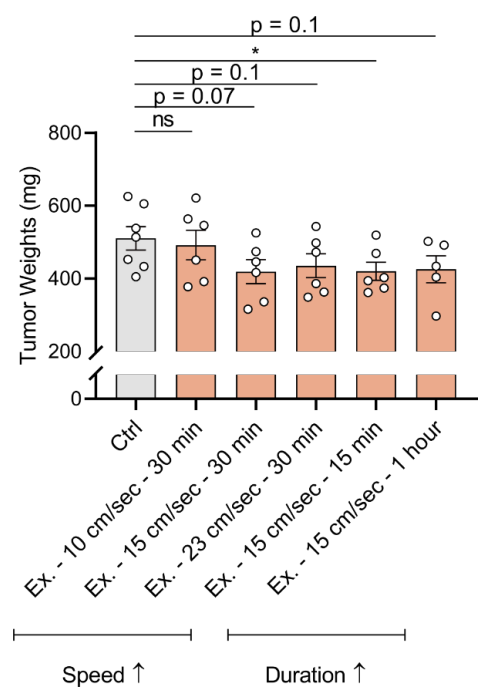

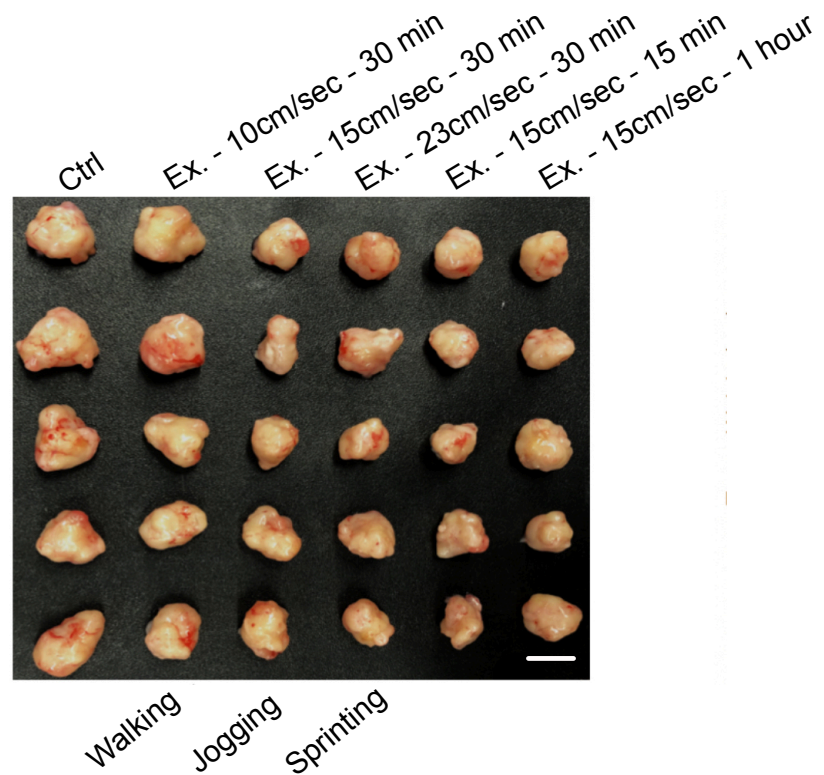

d

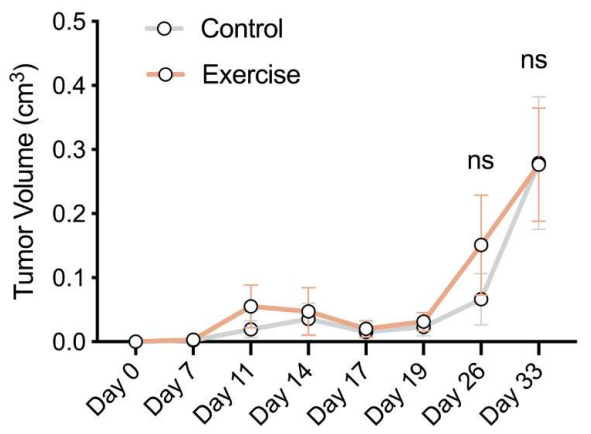

582 Extended Data Figure 1: Aerobic exercise restricts pancreatic cancer growth in a non-dose 583 dependent manner

584 a. 8-week old LSL-KRAS ${ }^{\mathrm{G} 12 \mathrm{D} / \mathrm{WT}}$; p48 ${ }^{\mathrm{Cre} / \mathrm{WT}}(\mathrm{KC})$ mice were exercised (Exercise) for 6 weeks.

585 Control mice remained sedentary. Mice were sacrificed at 14 weeks old. Histologic staining of 586 pancreata tissue sections with CK8 (red) and DAPI (blue) are shown. Quantification of the \% 
$587 \mathrm{CK} 8+$ of total DAPI positive cells per FOV was performed in ImageJ. Each dot represents 588 quantification in one field of view ( $8 \mathrm{FOV}, \mathrm{n}=3$ mice). Scale bar represents $200 \mu \mathrm{m}$. b. 8 -week 589 old female C57BL/6J wild type mice (WT) were injected orthotopically with $1 \times 10^{\wedge}$ cells of a 590 second independent KPC cell line, KPC 1203, into the pancreas at Day 0 and exercise was started 591 at Day 1. Control mice remained sedentary. Mice were sacrificed at Day 21 and tumors were 592 harvested. Representative tumor images (left) and quantification of tumor weights (right) are 593 shown. Scale bar represents $1 \mathrm{~cm}$. Each dot represents one tumor $(\mathrm{n}=6-7)$. c. 8 -week old female 594 C57BL/6J wild type mice (WT) were injected orthotopically with $1 \times 10^{\wedge 5} \mathrm{KPC} 4662$ cells into the 595 pancreas at Day 0 and specified exercise regimen was started at Day 1. Control mice remained 596 sedentary. Mice were sacrificed at Day 21 and tumors were harvested. Representative tumor 597 images (right) and quantification of tumor weights (left) are shown. Scale bar represents $1 \mathrm{~cm}$. 598 Each dot represents one tumor $(\mathrm{n}=5)$. d. 8 week old WT mice were injected subcutaneously with $5991 \times 10^{\wedge} \mathrm{KPC}$ FC1242 cells at Day 0 and exercise was started at Day 1. Control mice remained 600 sedentary. Tumor volumes were measured using calipers at 3-7 day intervals. Each dot represents 601 an average of five mice in each arm. ( $p>0.05=n s, p<0.05=*, p<0.001=* * *)$.

602

603

604

605

606

607

608

609

610

611

612

613

614

615

616 
Extended Data Figure 2

a

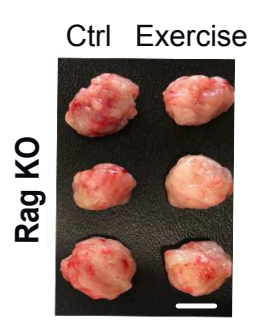

b

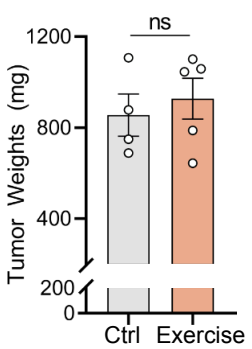

Cluster $1 \mathrm{CD} 8+\mathrm{T}$ cells

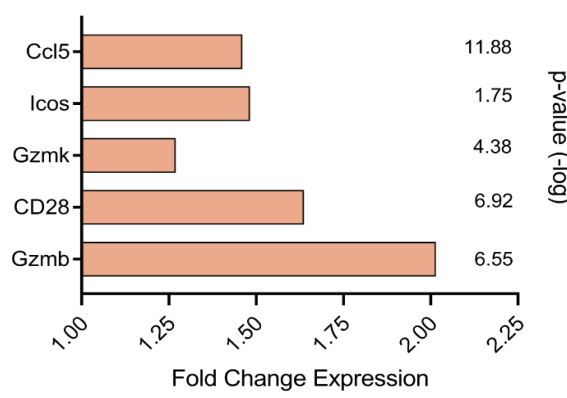

C
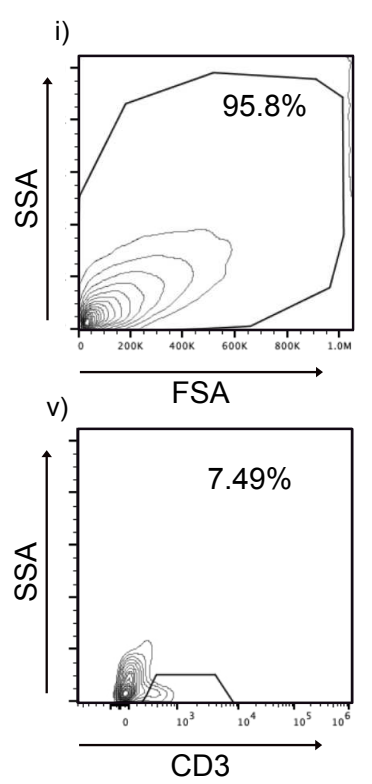

ii)
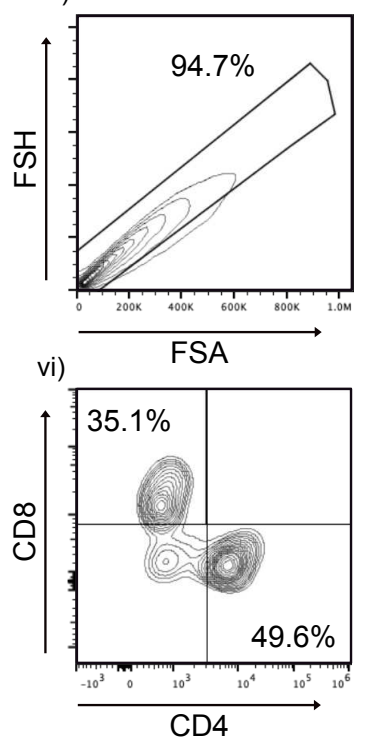

iii)

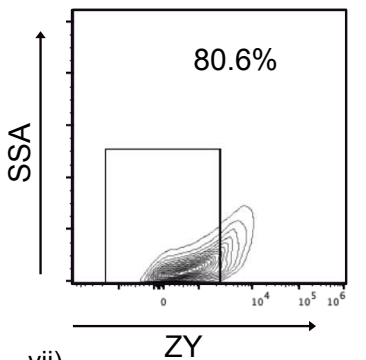

vii)

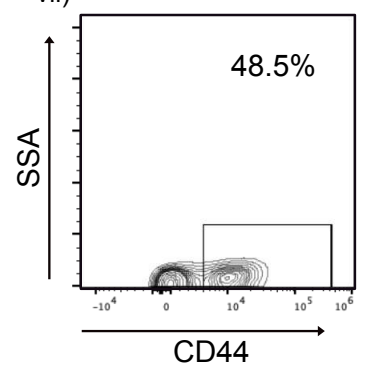

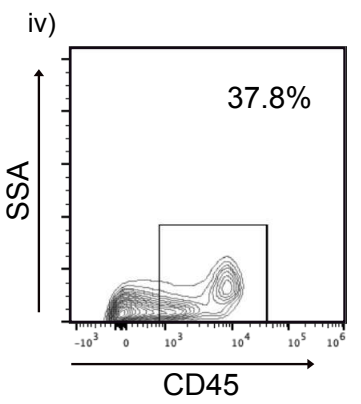

d
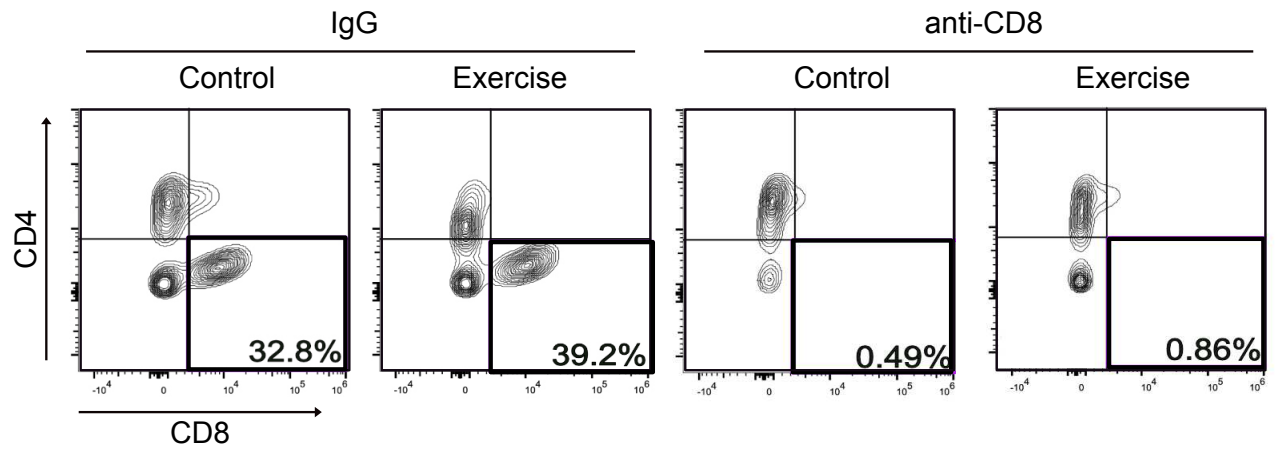

617

618 Extended Data Figure 2: Exercise-mediated tumor protection is CD8 T cell dependent

619 a. 8 week old male RAG1KO mice were injected orthotopically with $1 \times 10^{\wedge 5} \mathrm{KPC} 4662$ cells at

620 Day 0 and exercise was started at Day 1. Control mice remained sedentary. Mice were sacrificed 621 at Day 21. Representative tumor images (left) and quantification of tumor weights (right) are 622 shown. Scale bar represents $1 \mathrm{~cm}$. Each dot represents one tumor $(\mathrm{n}=4-5)$. b. Gene expression 
623 analysis of $\mathrm{CD} 8+\mathrm{T}$ cells, Cluster 1 from scRNAseq of exercised tumors showing fold change of 624 expression levels of $\mathrm{Ccl} 5$, Icos, Gzmk, Cd28, and Gzmb, relative to cells in other clusters. c. 625 Representative contour plots indicating example gating strategy used for flow cytometry analysis, 626 from top left: i) all cells in frame, ii) double-singlet discrimination, iii) live cells by zombie yellow 627 (ZY) negative, iv) all immune cells (CD45+), v) T cells (CD3+), vi) CD8+ T cells (CD4 negative, 628 CD8 positive, upper left quadrant), vii) expression level of CD44. d. Control and exercise mice 629 were treated $3 \mathrm{x} /$ week with isotype or $200 \mu \mathrm{g}$ of $\alpha$-CD8 blocking antibody starting on Day 1 . Mice 630 were sacrificed at Day 21. Single cell suspension derived from tumors were assessed by flow 631 cytometry for CD3, CD4, and CD8. Representative contour plots are shown for CD8+ CD4- cells 632 as a fraction of total CD3 positive cells. $(\mathrm{p}>0.05=\mathrm{ns})$. 


\section{Extended Data Figure 3}

a
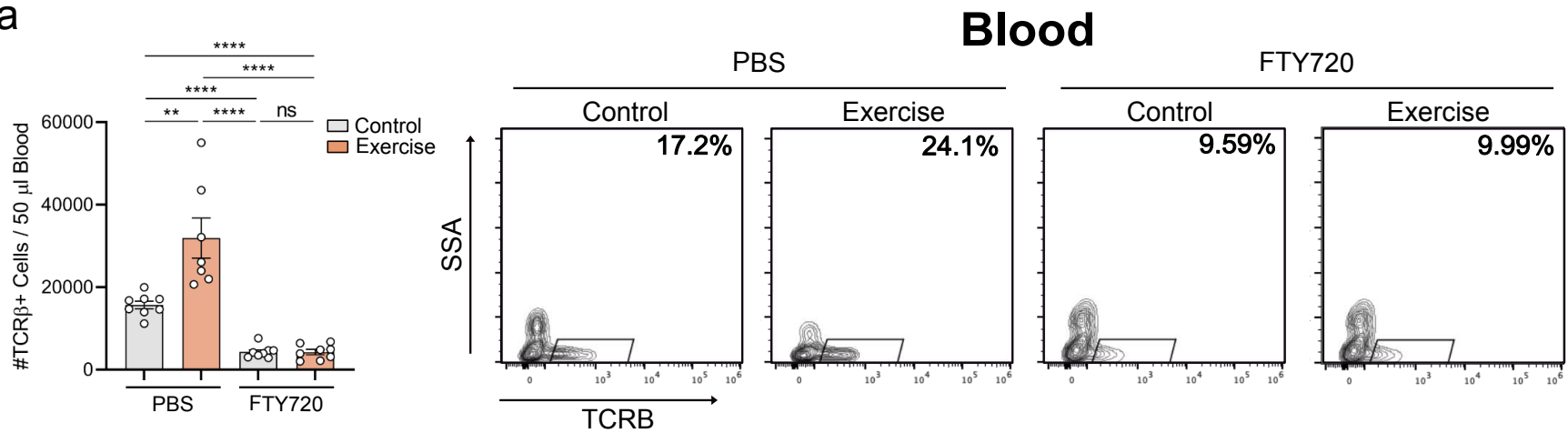

b
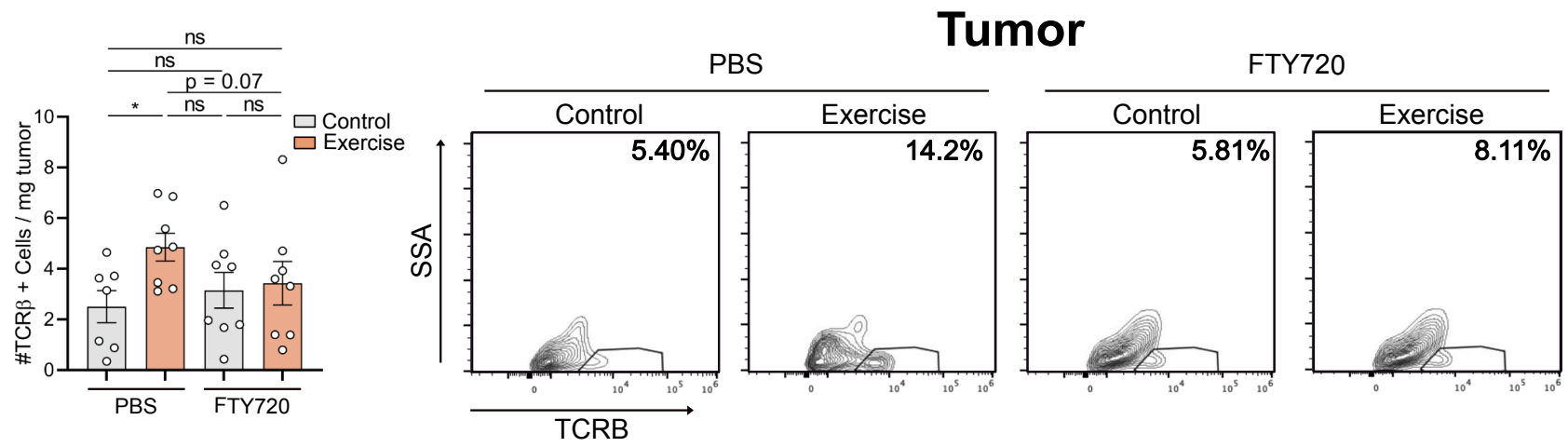

C
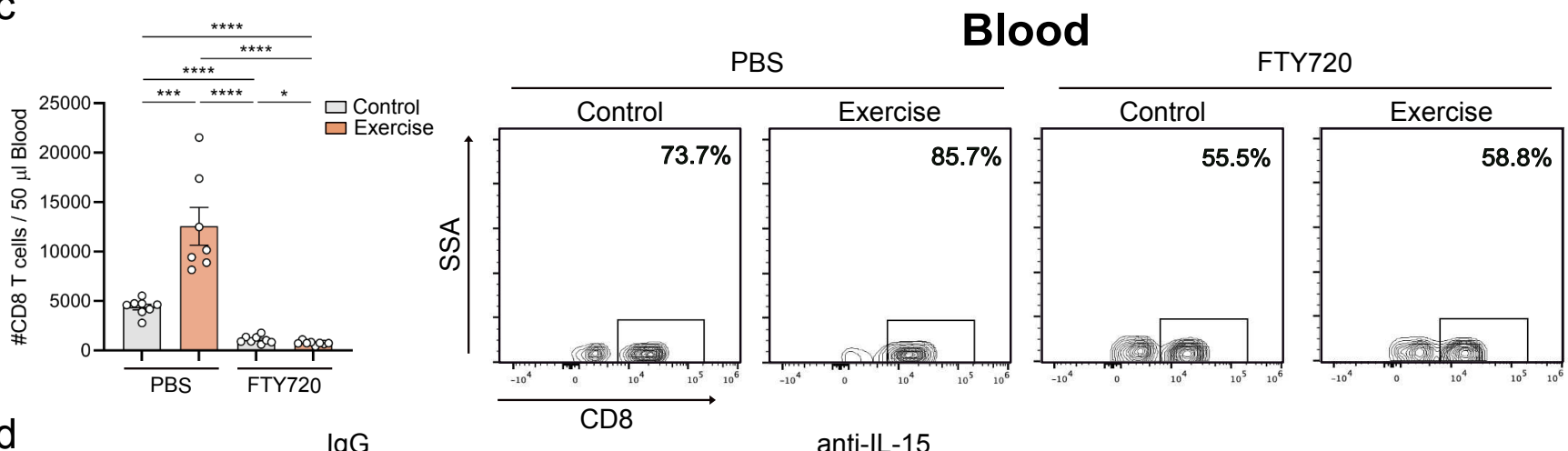

d

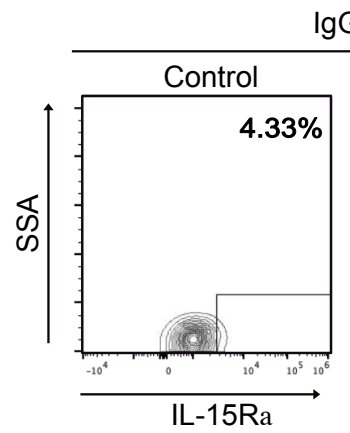

$\lg G$
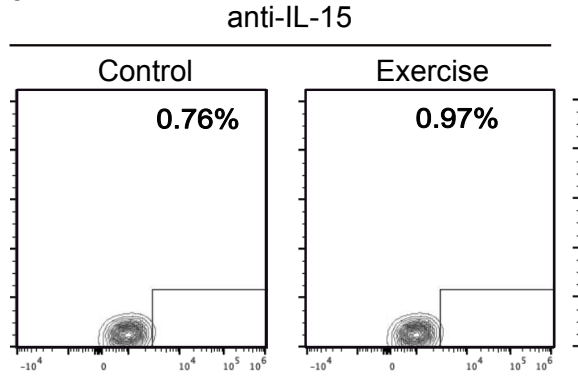

Isotype

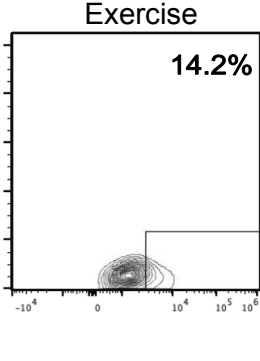

Blood

e
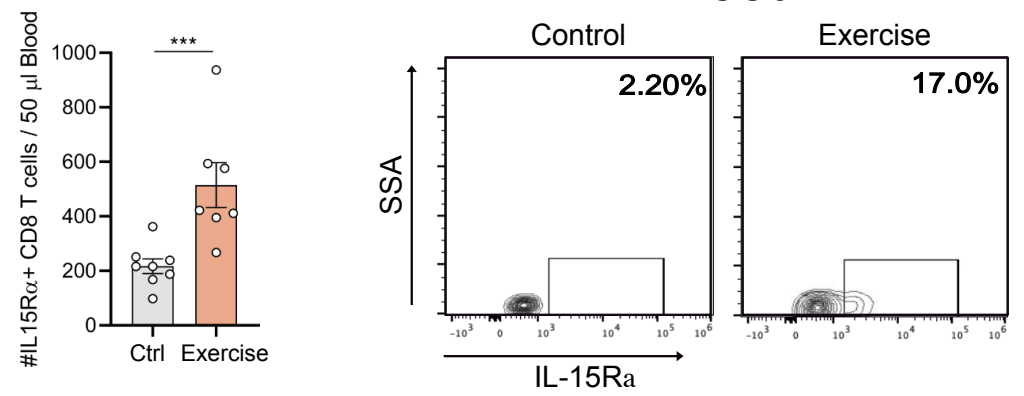


\section{IL15Ra+CD8 T cells are required for exercise-induced tumor protection}

a-c. 8 -week old female C57BL/6J wild type mice (WT) were injected orthotopically with $1 \times 10^{\wedge 5}$

KPC 4662 cells into the pancreas at Day 0 and exercise was started at Day 1. Control mice remained sedentary. Mice were treated daily either with PBS or $200 \mu$ of fingolimod (FTY720) starting on Day 1. Mice were sacrificed at Day 21. Single cell suspensions derived from whole blood or tumors were stained with antibodies against CD45, TCRB, CD4, and CD8, and analyzed by flow cytometry. Representative contour plots are shown for TCRB in blood (a), TCRB in tumor (b), and CD8 T cells in blood (c). Each dot represents one mouse ( $\mathrm{n}=7)$ d. 8 -week old female WT mice were injected orthotopically with $1 \times 10^{\wedge} \mathrm{KPC} 4662$ cells into the pancreas at Day 0 and exercise was started at Day 1. Control mice remained sedentary. Mice were treated 3x/week with isotype or $200 \mu \mathrm{g}$ of $\alpha$-IL15 neutralizing antibody starting on Day 1 post-op. Single cell suspensions derived from tumors were stained with antibodies against CD8 and IL-15R $\alpha$ and analyzed by flow cytometry. Representative contour plots for IL15R $\alpha+$ of total CD8+ cells, with staining isotype, are shown. e. 8-week old female WT mice were injected orthotopically with $1 \times 10^{\wedge 5} \mathrm{KPC} 4662$ cells into the pancreas at Day 0 and exercise was started at Day 1 . Control mice remained sedentary. Mice were sacrificed at Day 21, thirty minutes after completion of exercise and whole blood was harvested, digested, and assessed for relative numbers of IL15R $\alpha+$ CD8+ cells. Each dot represents one mouse $(n=7)$. Representative contour plots are shown. $(\mathrm{p}>0.05=$ ns, $\mathrm{p}<0.05=*, \mathrm{p}<0.01=* *, \mathrm{p}<0.001=* * *, \mathrm{p}<0.0001=* * * *)$. 


\section{Extended Data Figure 4}

a

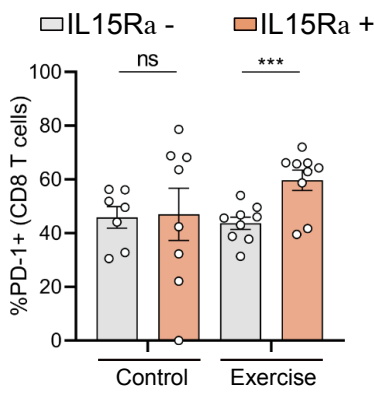

b
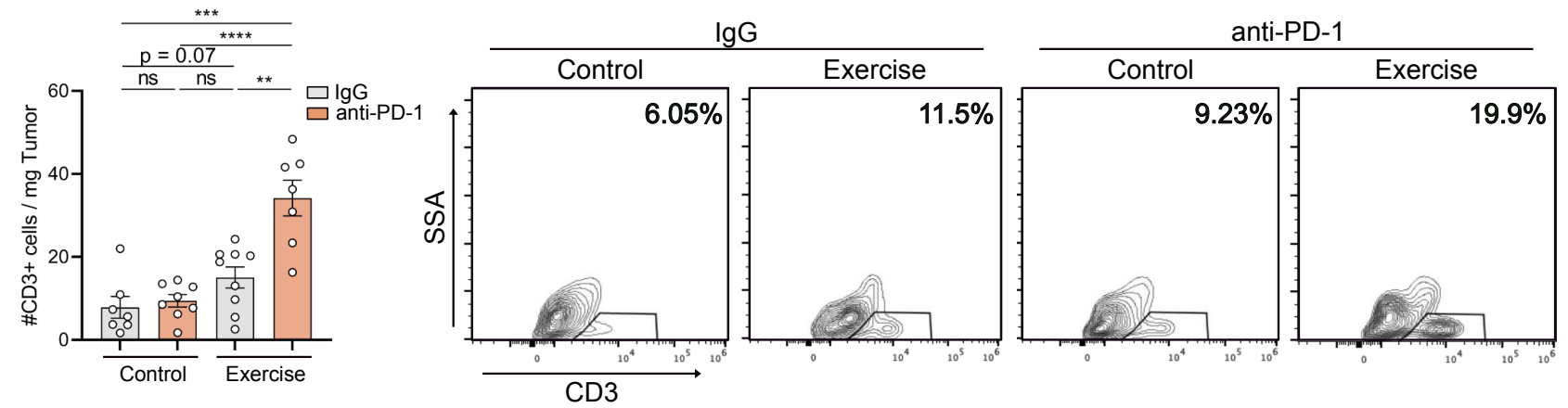

C

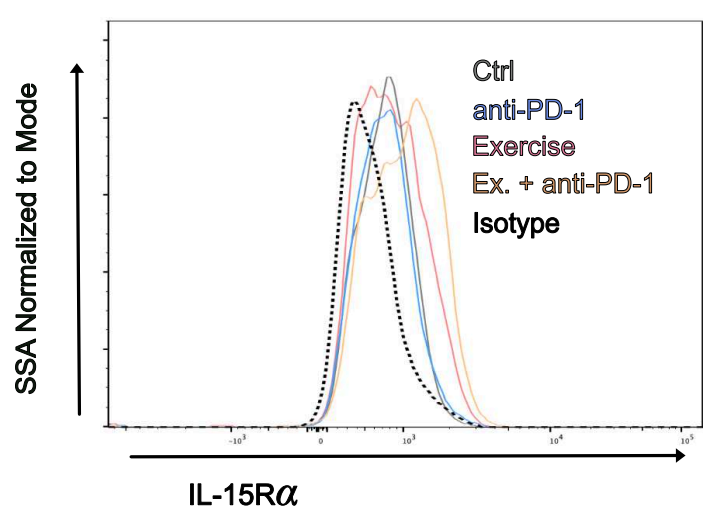

685

686 Extended Data Figure 4: Aerobic exercise and anti-PD-1 blockade exhibit intra-tumoral 687 immunological synergy

688 a. 8-week old female C57BL/6J wild type mice (WT) were injected orthotopically with $1 \times 10^{\wedge}$ 689 KPC 4662 cells into the pancreas at Day 0 and exercise was started at Day 1. Control mice 690 remained sedentary. Mice were sacrificed at Day 21 and tumors were harvested. Single cell 691 suspensions derived from tumors were stained with antibodies against CD3, CD8, PD-1 and IL-

$69215 \mathrm{R} \alpha$ and analyzed by flow cytometry. Each dot represents one tumor $(\mathrm{n}=7)$. b-c. 8-week old 693 female C57BL/6J wild type mice (WT) were injected orthotopically with $1 \times 10^{\wedge} 5$ KPC 4662 cells 694 into the pancreas at Day 0 and exercise was started at Day 1. Control mice remained sedentary. 
695 Mice were treated 3x/week with isotype or $200 \mu \mathrm{g}$ of $\alpha$-PD-1 blocking antibody starting on Day

696 3. Mice were sacrificed at Day 21. Single cell suspension derived from tumors were assessed by 697 flow cytometry for CD3 (b), CD8, and IL15R $\alpha+$ (c). Each dot represents one tumor (n=7-8) 698 Representative histogram plot is shown for IL15R $\alpha+$ in CD8 T cells. $(\mathrm{p}>0.05=\mathrm{ns}, \mathrm{p}<0.01=* *$, $699 \mathrm{p}<0.001=* * *, \mathrm{p}<0.0001=* * * *)$.

\section{$\underline{\text { References: }}$}

1. Brown JC, Winters-Stone K, Lee A, Schmitz KH. Cancer, physical activity, and exercise. Compr Physiol. 2012;2(4):2775-2809. doi:10.1002/cphy.c120005.

2. Kenfield, S. A., Stampfer, M. J., Giovannucci, E. \& Chan, J. M. Physical Activity and Survival After Prostate Cancer Diagnosis in the Health Professionals Follow-Up Study. J Clin Oncol 29, 726-732 (2011).

3. Schmid, D. \& Leitzmann, M. F. Association between physical activity and mortality among breast cancer and colorectal cancer survivors: a systematic review and meta-analysis. Ann Oncol 25, 12931311 (2014).

4. Liu, L. et al. Leisure time physical activity and cancer risk: evaluation of the WHO's recommendation based on 126 high-quality epidemiological studies. Brit J Sport Med 50, 372 (2016).

5. Jones, L. W. et al. Exercise and Prognosis on the Basis of Clinicopathologic and Molecular Features in Early-Stage Breast Cancer: The LACE and Pathways Studies. Cancer Res76, 5415-5422 (2016).

6. Rundqvist, H. et al. Cytotoxic T-cells mediate exercise-induced reductions in tumor growth. Elife 9, e59996 (2020).

7. Schadler, K. L. et al. Tumor vessel normalization after aerobic exercise enhances chemotherapeutic efficacy. Oncotarget 7, 65429-65440 (2016). 
8. Bedoya, C. A. F. et al. Exercise during preoperative therapy increases tumor vascularity in pancreatic tumor patients. Sci Rep-uk 9, 13966 (2019).

9. Pedersen, L. et al. Voluntary Running Suppresses Tumor Growth through Epinephrine- and IL-6Dependent NK Cell Mobilization and Redistribution. Cell Metab 23, 554-562 (2016).

10. Ashcraft, K. A., Peace, R. M., Betof, A. S., Dewhirst, M. W. \& Jones, L. W. Efficacy and Mechanisms of Aerobic Exercise on Cancer Initiation, Progression, and Metastasis: A Critical Systematic Review of In Vivo Preclinical Data. Cancer Res76, 4032-4050 (2016).

11. Estruel-Amades, S., Camps-Bossacoma, M., Massot-Cladera, M., Pérez-Cano, F. J. \& Castell, M. Alterations in the innate immune system due to exhausting exercise in intensively trained rats. Sci Rep-uk 10, 967 (2020).

12. Tseng, W. W. et al. Development of an Orthotopic Model of Invasive Pancreatic Cancer in an Immunocompetent Murine Host. Clin Cancer Res 16, 3684-3695 (2010).

13. Campbell, J. P. \& Turner, J. E. Debunking the Myth of Exercise-Induced Immune Suppression: Redefining the Impact of Exercise on Immunological Health Across the Lifespan. Front Immunol 9, 648 (2018).

14. Shek, P., Sabiston, B., Buguet, A. \& Radomski, M. Strenuous Exercise and Immunological Changes. Int J Sports Med 16, 466-474 (1995).

15. Bayne, L. J. et al. Tumor-Derived Granulocyte-Macrophage Colony-Stimulating Factor Regulates Myeloid Inflammation and T Cell Immunity in Pancreatic Cancer. Cancer Cell 21, 822-835 (2012).

16. Siret, C. et al. Deciphering the Crosstalk Between Myeloid-Derived Suppressor Cells and Regulatory T Cells in Pancreatic Ductal Adenocarcinoma. Front Immunol 10, 3070 (2020). 
17. Pylayeva-Gupta, Y., Lee, K. E., Hajdu, C. H., Miller, G. \& Bar-Sagi, D. Oncogenic Kras-Induced GM-CSF Production Promotes the Development of Pancreatic Neoplasia. Cancer Cell 21, 836-847 (2012).

18. Krüger, K., Lechtermann, A., Fobker, M., Völker, K. \& Mooren, F. C. Exercise-induced redistribution of $\mathrm{T}$ lymphocytes is regulated by adrenergic mechanisms. Brain Behav Immun 22, 324-338 (2008).

19. Foster, N. K. et al. Leukocytosis of exercise: role of cardiac output and catecholamines. Journal of Applied Physiology, 18AD, (1986).

20. Dimitrov, S., Lange, T. \& Born, J. Selective Mobilization of Cytotoxic Leukocytes by Epinephrine. $J$ Immunol 184, 503-511 (2010).

21. Chiba, K. FTY720, a new class of immunomodulator, inhibits lymphocyte egress from secondary lymphoid tissues and thymus by agonistic activity at sphingosine 1-phosphate receptors. Pharmacol Therapeut 108, 308-319 (2005).

22. Ruane, D. et al. Lung dendritic cells induce migration of protective $\mathrm{T}$ cells to the gastrointestinal tractLung dendritic cells' intestinal homing. $J$ Exp Medicine 210, 1871-1888 (2013).

23. Chiba, K. FTY720, a Novel Immunosuppressant, Induces Sequestration of Circulating Mature Lymphocytes by Acceleration of Lymphocyte Homing in Rats. Journal of Immunology (1998).

24. Pedersen, B. K., Åkerström, T. C. A., Nielsen, A. R. \& Fischer, C. P. Role of myokines in exercise and metabolism. J Appl Physiol 103, 1093-1098 (2007).

25. Idorn, M. \& Hojman, P. Exercise-Dependent Regulation of NK Cells in Cancer Protection. Trends Mol Med 22, 565-577 (2016). 
26. Berard, M., Brandt, K., Paus, S. B. \& Tough, D. F. IL-15 Promotes the Survival of Naive and Memory Phenotype CD8+ T Cells. J Immunol 170, 5018-5026 (2003).

27. Lu, J. Interleukin 15 Promotes Antigen-independent in Vitro Expansion and Long-Term Survival of Antitumor Cytotoxic T Lymphocytes1. Clinical Cancer Research 8, 3877-3884 (2002).

28. Wu, J. et al. Skeletal muscle antagonizes antiviral CD8+ T cell exhaustion. Sci Adv 6, eaba3458 (2020).

29. Carrero, R. M. S. et al. IL-15 is a component of the inflammatory milieu in the tumor microenvironment promoting antitumor responses. Proc National Acad Sci116, 201814642 (2018).

30. Marschner, N. et al. Association of Disease Progression with Health-Related Quality of Life Among Adults with Breast, Lung, Pancreatic, and Colorectal Cancer. Jama Netw Open 3, e200643 (2020).

31. Yang, A. et al. Autophagy sustains pancreatic cancer growth through both cell autonomous and non-autonomous mechanisms. Cancer Discov 8, CD-17-0952 (2018).

32. Grasselly, C. et al. The Antitumor Activity of Combinations of Cytotoxic Chemotherapy and Immune Checkpoint Inhibitors Is Model-Dependent. Front Immunol9, 2100 (2018).

33. Meyer, M. A. \& DeNardo, D. G. Better Together: B7S1 Checkpoint Blockade Synergizes with antiPD1. Immunity 48, 621-623 (2018).

34. IL Li, H. Y. et al. The Tumor Microenvironment Regulates Sensitivity of Murine Lung Tumors to PD-1/PD-L1 Antibody Blockade. Cancer Immunol Res 5, 767-777 (2017).

35. Ramos-Perez, W. D., Fang, V., Escalante-Alcalde, D., Cammer, M. \& Schwab, S. R. A map of the distribution of sphingosine 1-phosphate in the spleen. Nat Immunol 16, 1245-1252 (2015). 
36. Pedersen, L. et al. Voluntary Running Suppresses Tumor Growth through Epinephrine- and IL-6Dependent NK Cell Mobilization and Redistribution. Cell Metab 23, 554-562 (2016).

37. Jaatinen, T. \& Laine, J. Isolation of Mononuclear Cells from Human Cord Blood by Ficoll-Paque Density Gradient. Curr Protoc Stem Cell Biology 1, 2A.1.1-2A.1.4 (2007).

38. Tuck, M. K. et al. Standard Operating Procedures for Serum and Plasma Collection: Early Detection Research Network Consensus Statement Standard Operating Procedure Integration Working Group. J Proteome Res 8, 113-117 (2009).

39. Butler, A., Hoffman, P., Smibert, P., Papalexi, E. \& Satija, R. Integrating single-cell transcriptomic data across different conditions, technologies, and species. Nat Biotechnol 36, 411-420 (2018).

40. Stuart, T. et al. Comprehensive Integration of Single-Cell Data. Cell 177, 1888-1902.e21 (2019). 


\section{Figures}

a

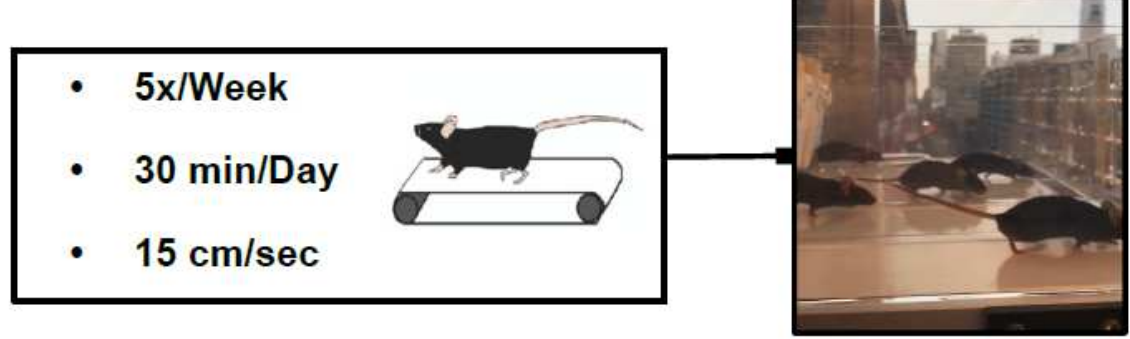

b
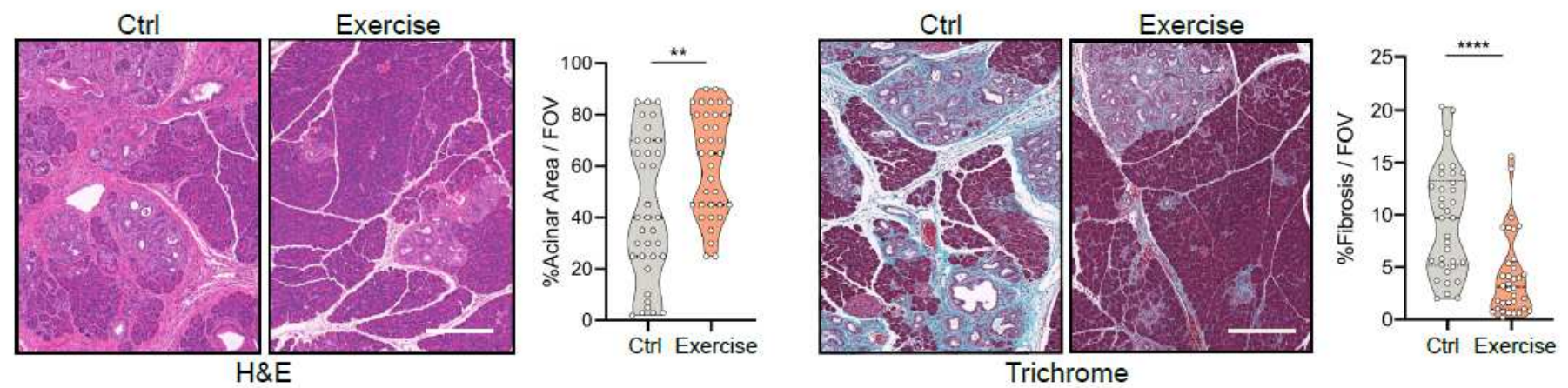

C

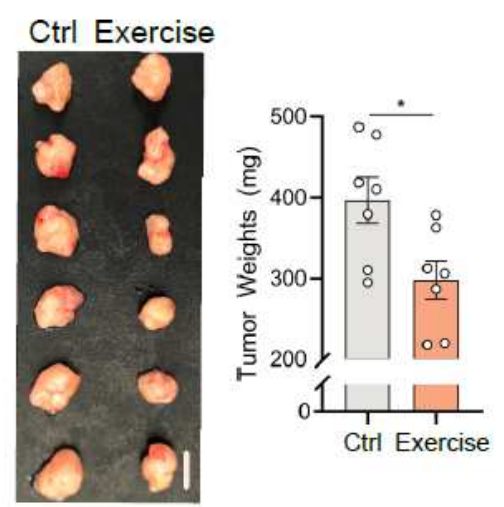

d
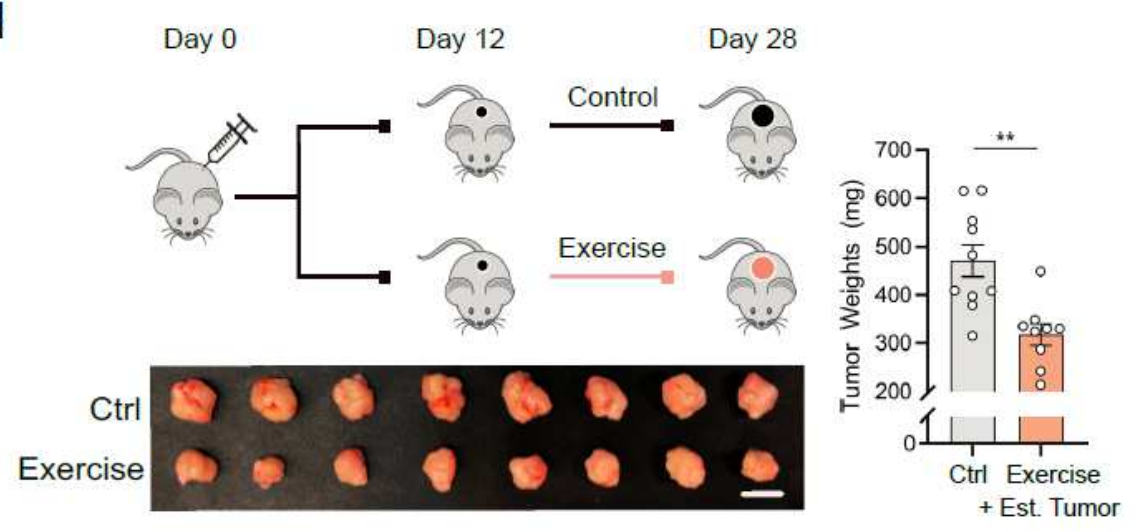

Figure 1

Aerobic exercise restricts pancreatic cancer growth and reduces tumor size a. The forced treadmill running model (aerobic exercise) is shown. The frequency, speed, and duration of aerobic exercise are indicated (left). Representative photograph of mice running on the treadmill is shown (right). b. 8-week old LSL-KRASG12D/WT; p48Cre/WT (KC) mice were exercised (Exercise) for 6 weeks. Control mice remained sedentary. Mice were sacrificed at 14 weeks old. Histologic staining of pancreata tissue sections with Hematoxylin and Eosin (H\&E, left) and Trichrome and Gomori (Trichrome, right) are shown. Representative images and quantification of acinar (left) and fibrotic (right) areas are shown. Each dot represents quantification in one field of view (FOV; 5-9 FOV analyzed from 3 tissue sections separated by at least $100 \mu \mathrm{m} ; \mathrm{n}=5 \mathrm{mice}$ ). Scale bar represents $200 \mu \mathrm{m}$. c. 8-week old female C57BL/6J wild type mice (WT) were injected orthotopically with 1x10^5 LSL-KRASG12D/WT; LSL-Trp53R172H/WT; p48Cre/WT (KPC) 4662 cells into the pancreas at Day 0 and exercise was started at Day 1. Control mice remained sedentary. Mice were sacrificed at Day 21. Representative tumor images (left) and quantification of tumor 
weights (right) are shown. Scale bar represents $1 \mathrm{~cm}$. Each dot represents one tumor $(n=7)$. d. 8- week old female WT mice were injected orthotopically with $5 \times 10^{\wedge} 4$ KPC 4662 cells into the pancreas at Day 0. At Day 12 following implantation, mice were randomized into control and exercise cohorts. Mice were sacrificed at Day 28. Schematic of experimental design (top), tumor images (bottom) and quantification of tumor weights (right) are shown. Scale bar represents $1 \mathrm{~cm}$. Each dot represents one tumor $(n=9-10)$. $(p<0.05=*, p<0.001=* *, p<0.0001=* * *)$.

a

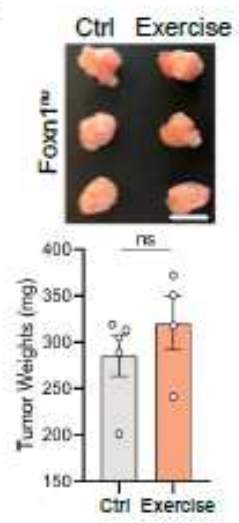

b
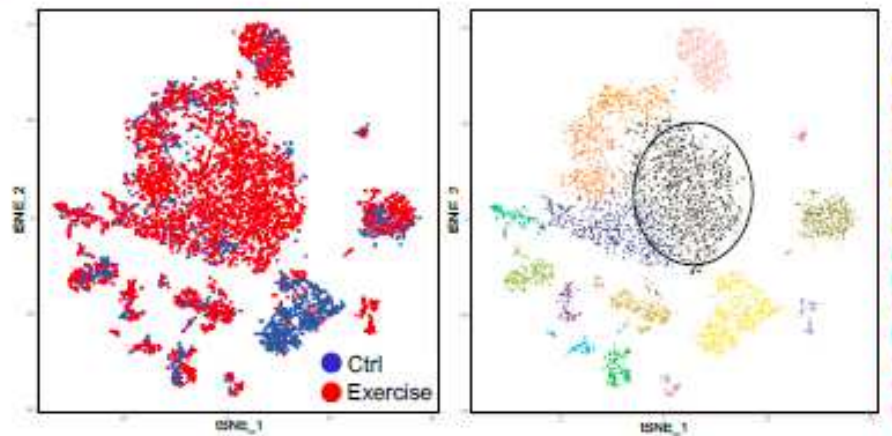

CD $8+T$ cells [1]

CD8 $+T$ cells [2]

Myeloid Suppressor [3]

CD 4+ T cells [4]

NK Cells [5]

$B$ cells $[6]$

CD4- CD8-T cells [7]

M2 Macrophages [8]

Dendritic Cells (P1)[9]

Monocyte/Macrophages [10]

Ductal/Tumor Cells [11]

- Dendritic Cells (L) [12]

CD209hi Myeloid [13]

Myeloid Suppressor [14]

Dendritic Cells (L2) [15]

C
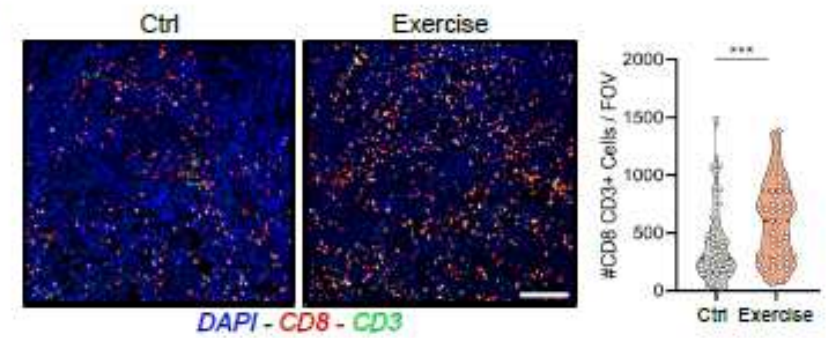

d

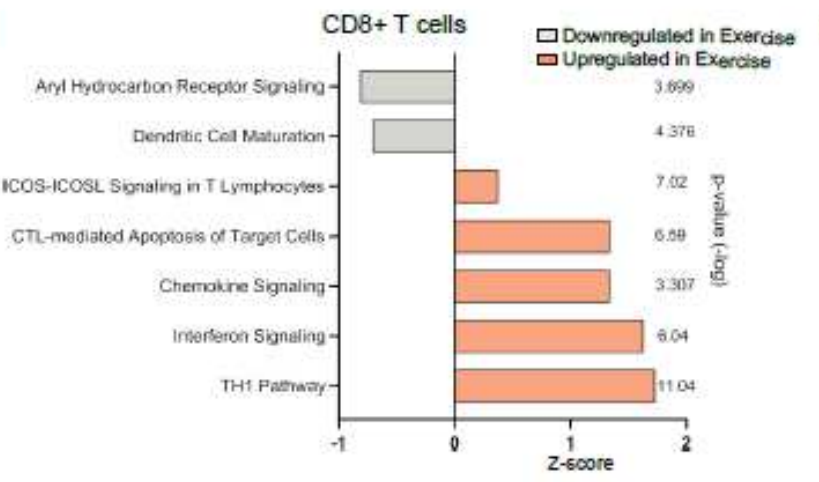

e

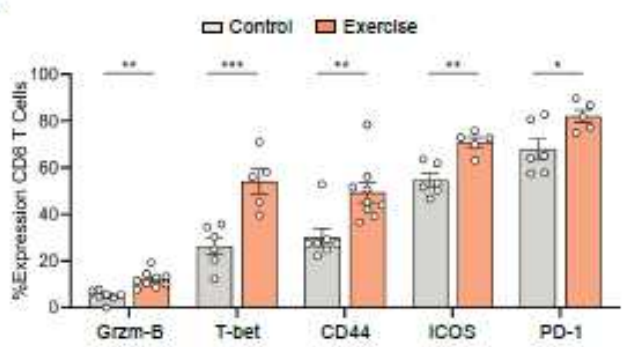

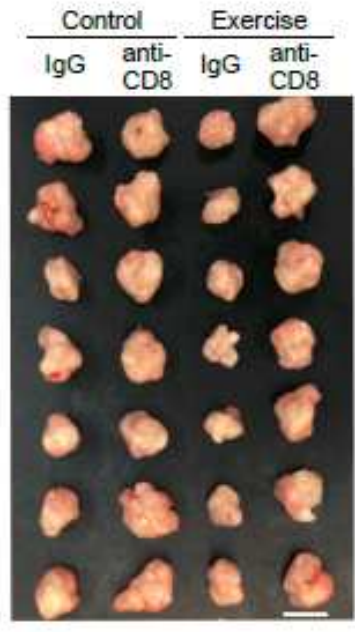

g
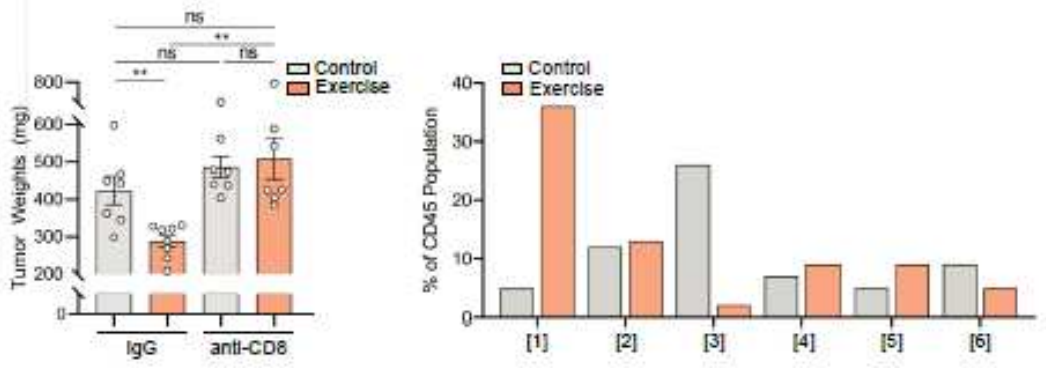

Figure 2 
The tumor protective effects of aerobic exercise are dependent on CD8 T cells a. 8 week old female athymic nude NU/J mice were injected orthotopically with $1 \times 10^{\wedge} 5 \mathrm{KPC} 4662$ cells at Day 0 and exercise was started at Day 1. Control mice remained sedentary. Mice were sacrificed at Day 21. Representative tumor images (top) and quantification of tumor weights (below) are shown. Scale bar represents $1 \mathrm{~cm}$. Each dot represents one tumor $(n=4-5)$. b-g. 8- week old female C57BL/6J wild type mice (WT) were injected orthotopically with $1 \times 10^{\wedge} 5 \mathrm{KPC} 4662$ cells into the pancreas at Day 0 and exercise was started at Day 1. Control mice remained sedentary. Mice were sacrificed at Day 21 and tumors analyzed as follows. b. Single cell RNASeq (scRNAseq) was performed on live leukocytes (PI-, CD45+) isolated by FACS. The distribution of cellular clusters was determined using the tSNE algorithm: control (blue) and exercise (red) leukocytes were identified (left), phenotypic clusters were identified by distinct colors (middle) and labeled (right) ( $\mathrm{n}=3$ tumors pooled in each group). c. Tumor sections were stained by multiplex immunofluorescence for CD8 (red), CD3 (green), and DAPI (blue). The number of CD8+ CD3+ (yellow) cells were quantified in ImageJ. Each dot represents quantification in one field of view (13-16 FOV analyzed; $n=4)$. Scale bar represents $75 \mu \mathrm{m}$. d. Upstream canonical pathway perturbations were derived using ingenuity pathway analysis. Pathways up-regulated (orange) or down-regulated (grey) in exercise were identified in the CD8+ T cell, Cluster 1. e. Single cell suspensions derived from tumors were stained with antibodies against CD45, CD3, CD4, CD8, Granzyme-B, T-bet, CD44, ICOS, and PD-1 and analyzed by flow cytometry. Each dot represents one tumor $(n=6)$. f. Control and exercise mice were treated $3 x /$ week with isotype or $200 \mu \mathrm{g}$ of a-CD8 blocking antibody starting on Day 1. Tumor images (left) and quantification of tumor weights (right) are shown. Scale bar represents $1 \mathrm{~cm}$. Each dot represents one tumor $(n=7)$. g. Quantification of the relative proportion of the six most prominent immune populations in control and exercise tumors from scRNAseq. $(p>0.05=n s, p<0.05=*, p<0.01=* \star, p<0.001=* \star \star)$. 
a

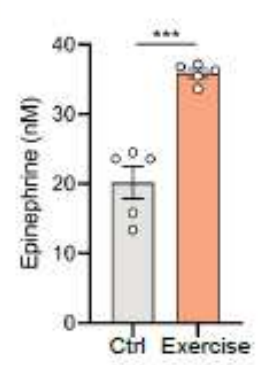

b

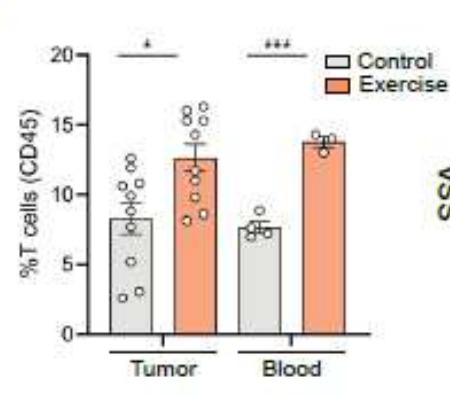

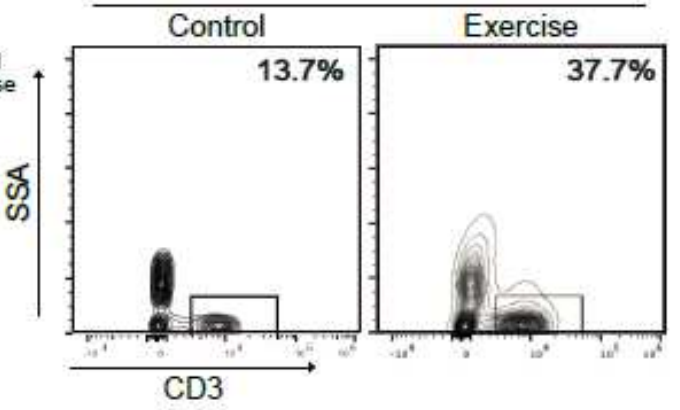

C

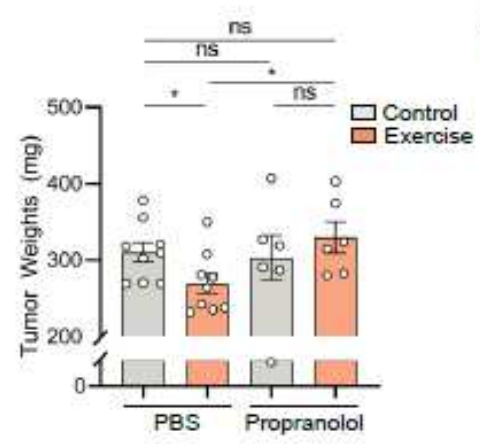

d
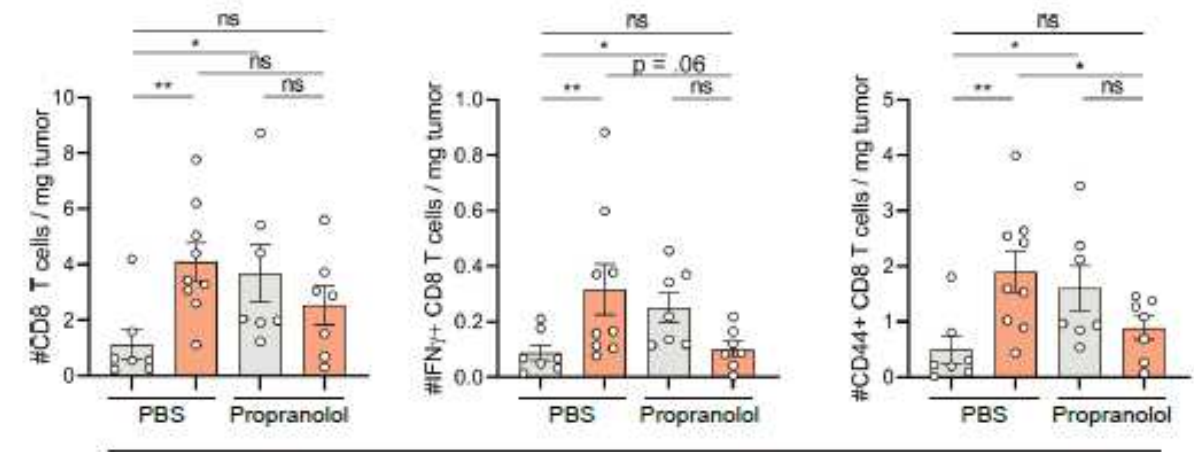

e
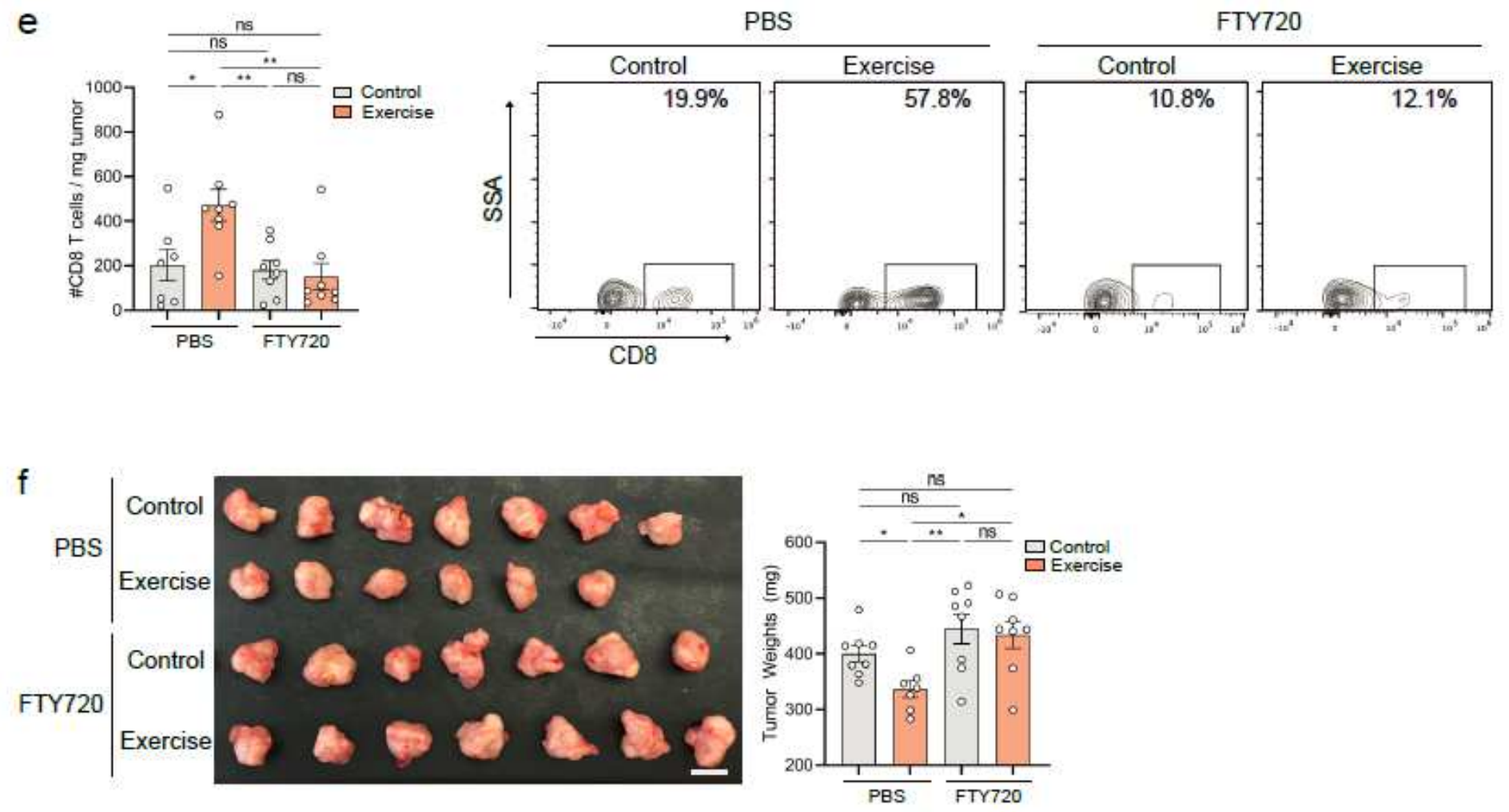

\section{Figure 3}

Exercise-induced increase in intra-tumoral CD8 T cells is $\beta$-adrenergic and S1P gradient dependent. a. 8 week-old female WT mice were exercised for 30 minutes at $15 \mathrm{~cm} / \mathrm{sec}$ and were sacrificed 20 minutes after completion of exercise. $200 \mu \mathrm{l}$ of whole blood was collected, sera were isolated using centrifugation, and samples were subjected to ELISA for detection of Epinephrine levels. Each dot represents one mouse $(n=5)$. b-f. 8-week old female C57BL/6J wild type mice (WT) were injected orthotopically with $1 \times 10^{\wedge} 5$ 
KPC 4662 cells into the pancreas at Day 0 and exercise was started at Day 1. Control mice remained sedentary. b. Mice were sacrificed at Day 21, thirty minutes after completion of exercise, and tumors and whole blood were harvested, digested, and assessed for relative numbers of CD3+ T cells. Each dot represents one mouse ( $n=3-4$ each for blood, $n=10$ for tumors). Representative contour plots are shown for CD3+ cells as a percentage of CD45+ cells in whole blood. c-d. Mice were provided with either normal drinking water or $.5 \mathrm{~g} / \mathrm{L}$ propranolol drinking water ad libitum starting on Day 1 . Mice were sacrificed on Day 21 and tumor weights were quantified (c), and single cell suspensions derived from tumors were stained with antibodies against CD45, CD3, CD8, IFNg, and CD44 and analyzed by flow cytometry (d). Each dot represents one tumor ( $n=6-9$ ). e-f. Mice were treated daily either with PBS or $200 \mu \mathrm{l}$ of fingolimod (FTY720) starting on Day 1. Mice were sacrificed at Day 21. Single cell suspensions derived from tumors were stained with antibodies against CD45, TCRB, CD4, and CD8, and analyzed by flow cytometry. Each dot represents one tumor. Representative flow cytometry plots are shown for CD8+ cells as fraction of CD4- CD3+ cells (e). Tumor images (left) and quantification of tumor weights (right) are shown (f). Scale bar represents $1 \mathrm{~cm}$. Each dot represents one tumor $(n=7) .(p>0.05=n s, p<0.05=*, p$ $<0.01=* \star, p<0.001=* \star *)$. 
a

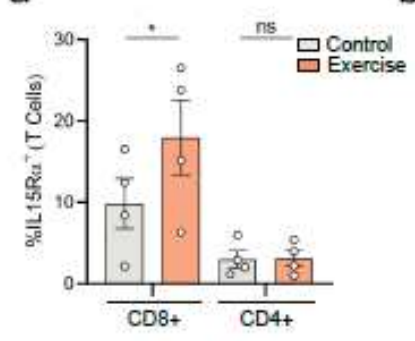

b

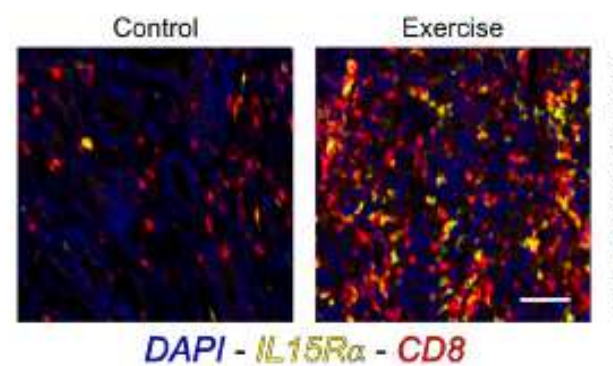

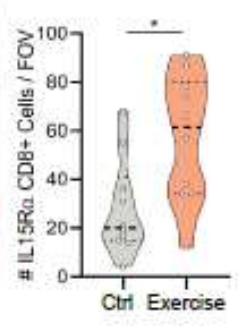

C
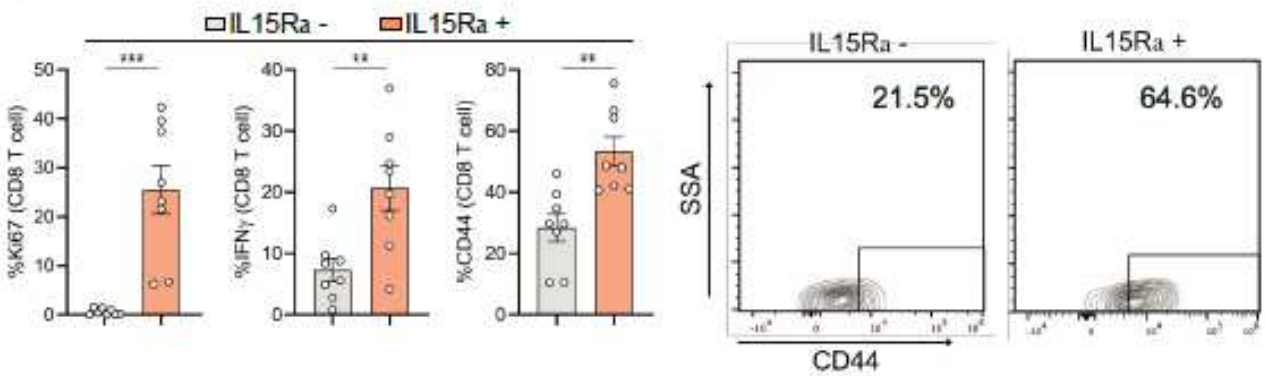

d

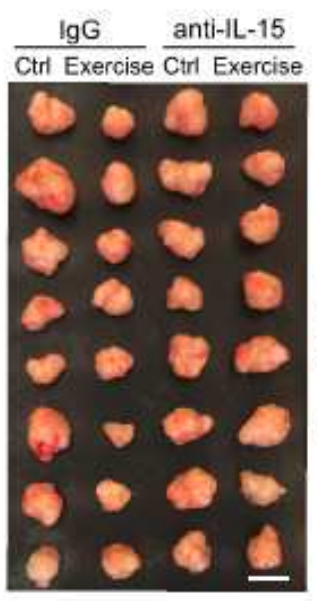

e

f
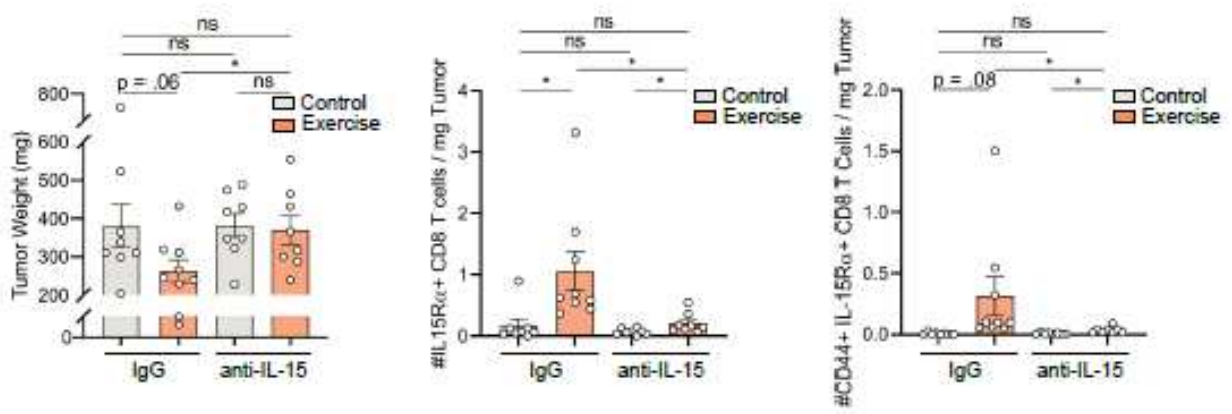

g
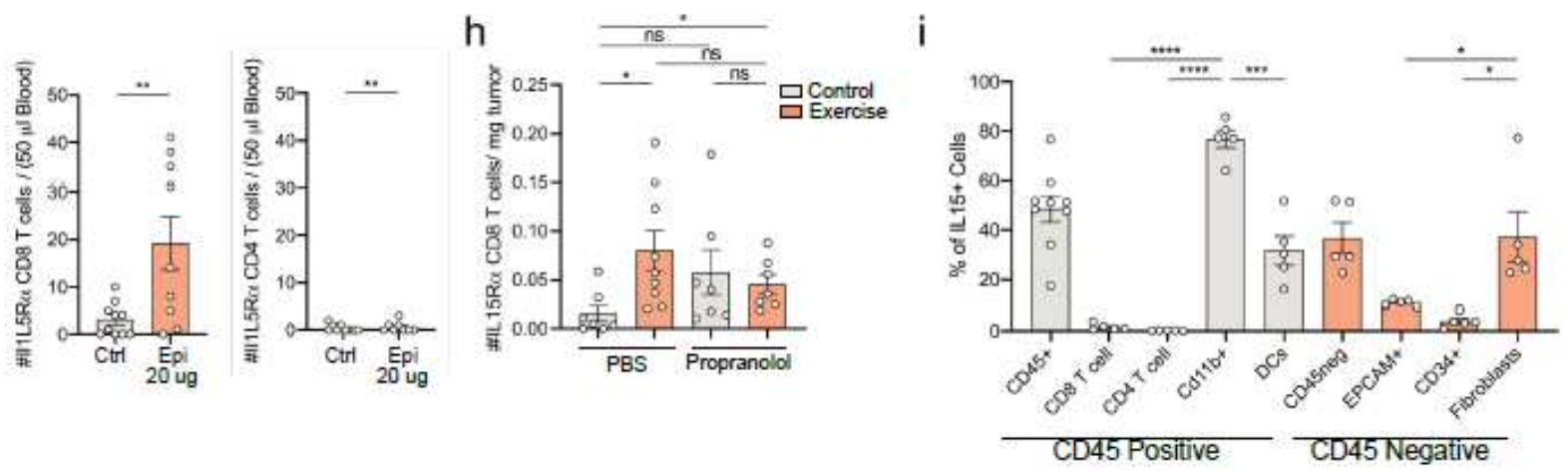

Figure 4

IL-15/IL15Ra axis is required for exercise-mediated tumor protection a-f. 8-week old female C57BL/6J wild type mice (WT) were injected orthotopically with $1 \times 10^{\wedge} 5 \mathrm{KPC} 4662$ cells into the pancreas at Day 0 and exercise was started at Day 1. Control mice remained sedentary. Mice were sacrificed at Day 21 and tumors analyzed as follows. a. Single cell suspensions derived from tumors were stained with antibodies against CD45, CD3, CD4, CD8, and IL-15Ra and analyzed by flow cytometry. Each dot represents one 
tumor $(n=4)$. b. Tumor sections were stained by multiplex immunofluorescence for CD8 (red), IL-15Ra (yellow), and DAPI (blue). The number of CD8+ IL-15Ra+ (orange) cells were quantified in ImageJ. Each dot represents quantification per field of view (3-4 FOV analyzed; $n=3$ ). Scale bar represents $40 \mu \mathrm{m}$. c. Single cell suspensions derived from tumors were stained with antibodies against CD8, Ki67, IFNg, CD44 and IL-15Ra and analyzed by flow cytometry. Each dot represents one tumor $(n=8)$. Representative flow cytometry plots are shown for CD44. d-f. Mice were treated 3x/week with isotype or $200 \mu \mathrm{g}$ of a-IL15 neutralizing antibody starting on Day 1 post-op. Tumor images (left) and quantification of tumor weights (right) are shown. Scale bar represents $1 \mathrm{~cm}(\mathrm{~d})$. Single cell suspensions derived from tumors were stained and analyzed by flow cytometry for number of IL-15Ra+CD8+ T cells (e) and CD44+ IL-15Ra+ CD8+ T cells (f). Each dot represents one tumor ( $n=8-9)$. g. 9-week-old female WT mice were injected i.p. with PBS or $20 \mu \mathrm{g}$ of Epinephrine. Thirty minutes post-treatment $100 \mu \mathrm{l}$ of whole blood was isolated and assessed by flow cytometry for number of IL-15Ra+ CD8+ T cells (left) or IL-15Ra+ CD4+ T cells (right). Each dot represents one mouse $(n=9-10)$. h. 8-week old female C57BL/6J wild type mice (WT) were injected orthotopically with $1 \times 10^{\wedge} 5 \mathrm{KPC} 4662$ cells into the pancreas at Day 0 and exercise was started at Day 1. Control mice remained sedentary. Mice were provided either normal drinking water or . $5 \mathrm{~g} / \mathrm{L}$ propranolol drinking water ad libitum starting on Day 1. Mice were sacrificed at Day 21. Single cell suspensions derived from tumors were assessed by flow cytometry for number of IL-15Ra+ CD8+T cells. Each dot represents one tumor $(n=6-9)$. i. 8-week old female C57BL/6J wild type mice (WT) were injected orthotopically with $1 \times 10^{\wedge} 5 \mathrm{KPC} 4662$ cells into the pancreas at Day 0 . All mice remained sedentary. Single cell suspensions derived from tumors were stained with antibodies against CD45, CD3, CD8 T cells (CD8+ CD3+), CD4 T cells (CD4+ CD3+), CD11b, DCs (CD11c+ MHCIl hi), EPCAM, CD34, Fibroblasts (CD140a+ CD34- EPCAM CD45-) and analyzed by flow cytometry for the expression of IL-15. Each dot represents one tumor $(n=5-9) .(p>0.05=n s, p<0.05=*, p<0.01=* \star, p<0.001=\star \star \star, p<0.0001=\star \star \star \star)$. 
a

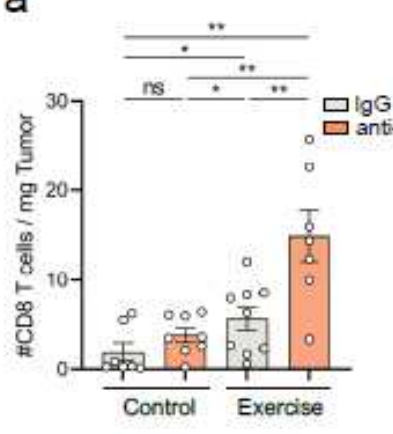

b

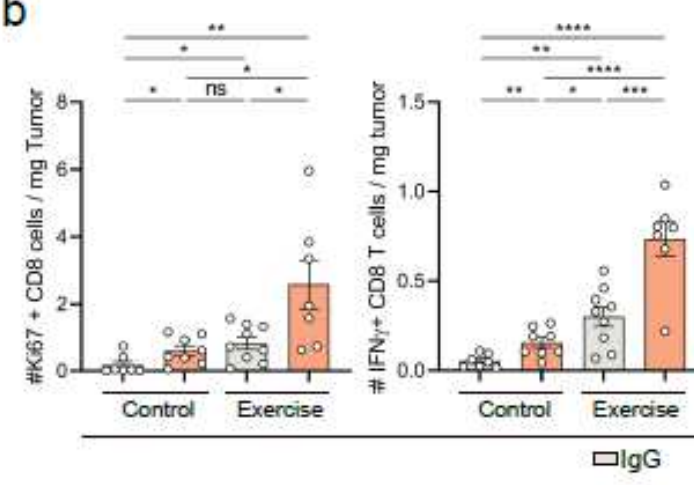

$\lg G$

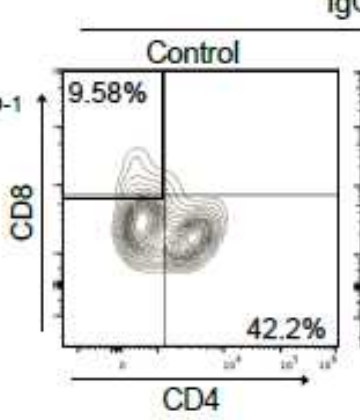

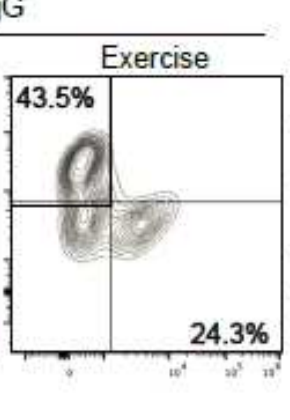

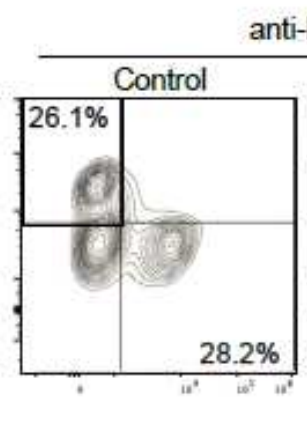

anti-PD1

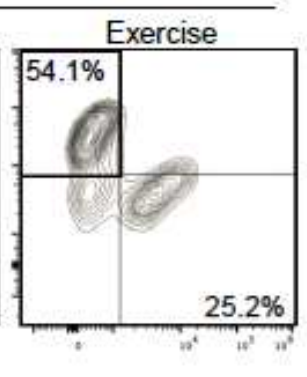

C

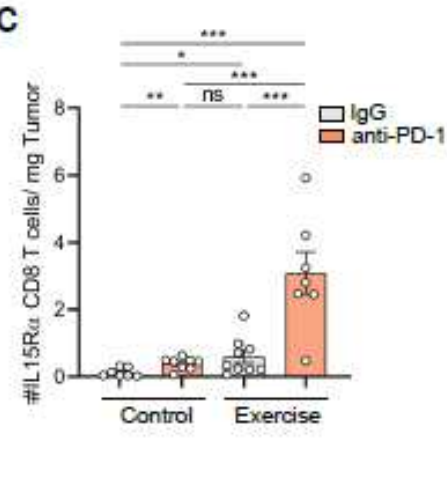

d
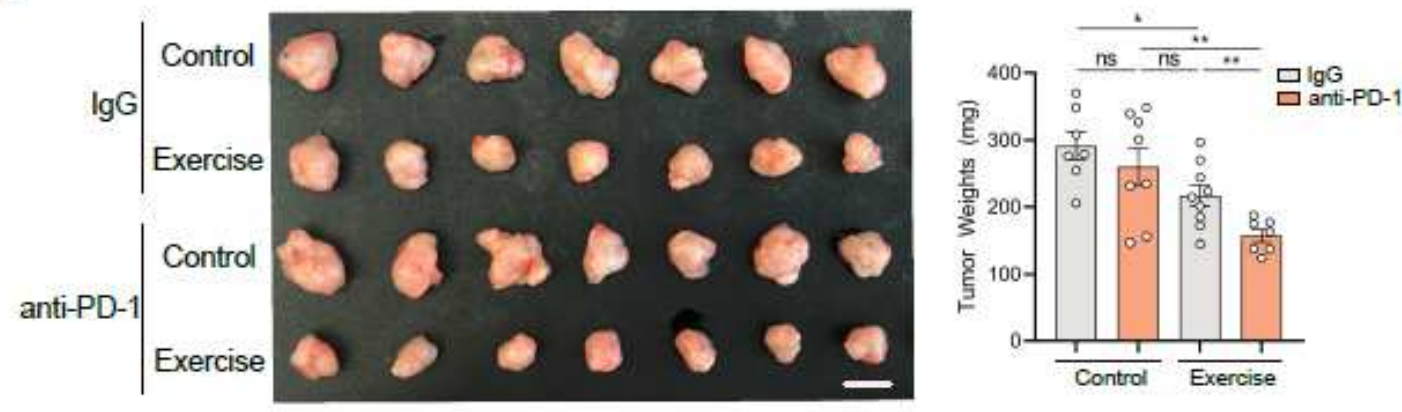

e

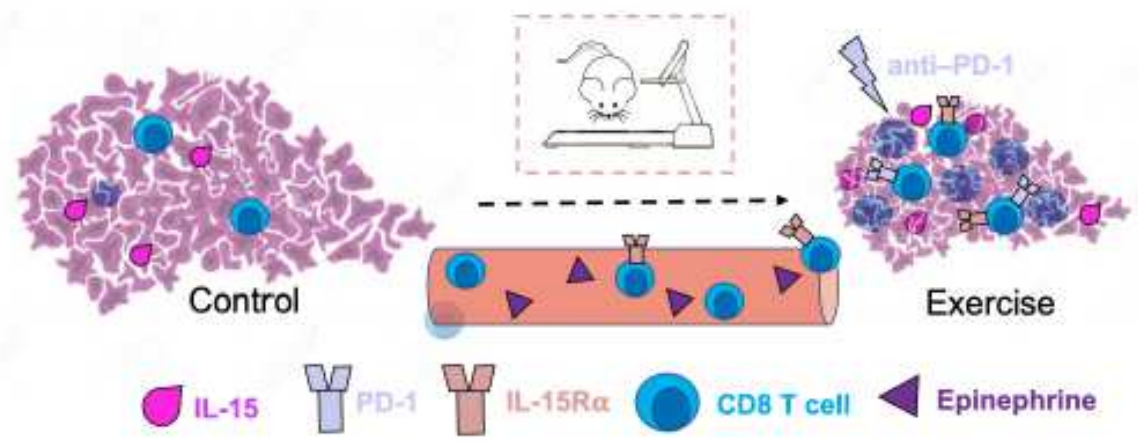

\section{Figure 5}

Aerobic Exercises Sensitizes Pancreatic Cancer to anti-PD1 Immunotherapy a-d. 8-week old female C57BL/6J wild type mice (WT) were injected orthotopically with $1 \times 10^{\wedge} 5 \mathrm{KPC} 4662$ cells into the pancreas at Day 0 and exercise was started at Day 1. Control mice remained sedentary. Mice were treated $3 x /$ week with isotype or $200 \mu \mathrm{g}$ of a-PD-1 blocking antibody starting on Day 3. Mice were sacrificed at Day 21. Single cell suspensions derived from tumors were assessed by flow cytometry for CD3, CD4, and CD8 (a), 
for Ki67, Granzyme-B, T bet, and IFNg expression on CD8 T cells (b) and number of IL-15Ra+ CD8+ T cells (c). Tumor images (left) and quantification of tumor weights (right) are shown (d). Scale bar represents 1 $\mathrm{cm}$. Each dot represents one tumor $(n=7-8)$. e. Schematic depicting proposed mechanism for the impact of aerobic exercise on CD8 T cell mobilization, pancreatic tumor growth and anti-tumor immunity. $(p>$ $0.05=n s, p<0.05=*, p<0.01=* \star, p<0.001=* \star \star, p<0.0001=* \star \star *)$. 